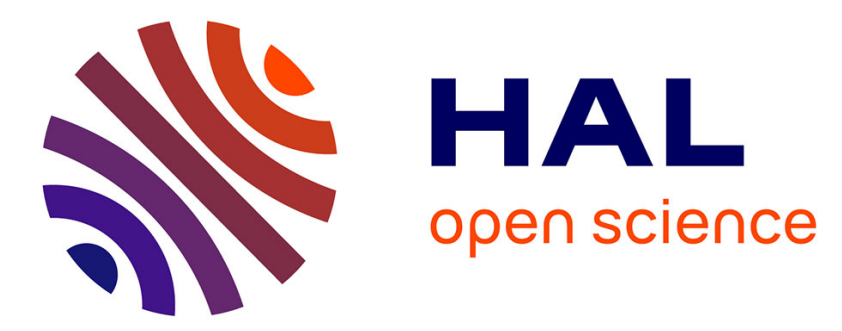

\title{
Polar and Cartesian Structure in the Data of Fitts's (1954) Classic Experiments-With a Criterion for Distinguishing a Strong and a Weak Version of Fitts' Law \\ Yves Guiard
}

\section{To cite this version:}

Yves Guiard. Polar and Cartesian Structure in the Data of Fitts's (1954) Classic Experiments-With a Criterion for Distinguishing a Strong and a Weak Version of Fitts' Law. Journal of Motor Behavior, In press. hal-02081613

\section{HAL Id: hal-02081613 https://hal.science/hal-02081613}

Submitted on 27 Mar 2019

HAL is a multi-disciplinary open access archive for the deposit and dissemination of scientific research documents, whether they are published or not. The documents may come from teaching and research institutions in France or abroad, or from public or private research centers.
L'archive ouverte pluridisciplinaire HAL, est destinée au dépôt et à la diffusion de documents scientifiques de niveau recherche, publiés ou non, émanant des établissements d'enseignement et de recherche français ou étrangers, des laboratoires publics ou privés. 
Polar and Cartesian Structure in the Data of Fitts's (1954) Classic Experiments-With a Criterion for Distinguishing a Strong and a Weak Version of Fitts' Law

Pre-print of an article to appear in the Journal of Motor Behavior.

\author{
Yves Guiard \\ yves.guiard@telecom-paristech.fr
}

LTCI, Telecom ParisTech, Université Paris-Saclay, Paris, France

LRI, Université Paris-Sud, CNRS, Université Paris-Saclay, Orsay, France 


\begin{abstract}
Fitts' law says that $T$, the time it takes to reach a target of width $W$ located at distance $D$, varies as a logarithmic or power function of the quotient of $D / W$. The received, strong version of the law requires isochrony-namely, the invariance of $T$ across different $D / W$ conditions with the same quotient. However, there is room for a weaker, yet nontrivial version of Fitts' law where the effect of scale does show up while combining in an additive way with that of the crucial quotient.

The paper revisits Fitts's (1954) classic experiments in light of Cartesian/polar analysis (Guiard, 2009) and reports intriguing new results. The strong, isochronous version of Fitts' law holds true for tapping, but contrary to a widely-held view the other two experiments were not just half-failed corroborations. While the disc-transfer data eloquently illustrate the weak, nonisochronous version of Fitts' law, the pin-transfer data definitely violate the law by showing a strong interaction between scale and the quotient of $D / W$. Surprisingly, however, the results of the pin-transfer experiment are remarkably simple in the alternative Cartesian description system, as $D$ and $W$ exerted separate, additive effects on $T$.
\end{abstract}




\section{Public Significance Statement}

Sixty years ago Fitts published the first experimental demonstration of the quantitative rule famously known today as Fitts' law. This paper reports a complete reanalysis of the numerical data that Fitts tabulated in detail in his article, revealing patterns of remarkable coherence that had been so far unsuspected due to undetected ambiguities concerning the dimensions of Fitts' law. One particular intriguing discovery is that there exist two different versions of Fitts' law, both eloquently illustrated by Fitts's own data.

\section{Keywords}

Fitts' law; aimed movement; Cartesian/polar analysis; scale; isochrony 
In a famous article ${ }^{1}$ Fitts (1954) reported the first experimental demonstration that in general the time $T$ needed to reach a target of width $W$ located at distance $D$ varies about linearly with the logarithm of the quotient of the division of $D$ by $W$. This simple quantitative rule of thumb, famously known today as Fitts' law, has been abundantly praised for its robustness and generality (e.g., Kelso, 1992; Meyer, Abrams, Kornblum, Wright, \& Smith, 1988; Soukoreff \& MacKenzie, 2004). Ever since Fitts (1954), however, there has been considerable debate about the exact mathematical description of the rule (e.g., Guiard \& Olafsdottir, 2011; Hoffmann, 2013; Kvalseth, 1980; MacKenzie, 1992) as well as about its substantive theoretical explanation (e.g., Crossman \& Goodeve, 1983; Guiard \& Rioul, 2015; Meyer, Abrams, Kornblum, Wright, \& Smith,1988; Plamondon \& Alimi, 1997; Schmidt, Zelaznik, Hawkins, Frank, \& Quinn, 1979).

It should be noted that the present paper is not about the possible explanatory mechanisms of Fitts' law, nor about the comparative merits of the various mathematical functions that can be conjured up to formulate the rule. Our focus here is the basic structure of the formulation, which more often than not consists of a mathematical function of the form $T=f(x)$, where movement time $T$ is made to depend on a single independent variable $x$-typically some index of difficulty computed from the dimensionless quotient of $D / W$. The paper argues that this description of Fitts' law data is logically problematic given that the factorial design needed to obtain Fitts' law data requires the orthogonal manipulation of two factors. Since the question asked in any Fitts' law experiment involves two degrees of freedom (DoF), so should the answer-i.e., the description of the data. Thus the statement of Fitts' law should be of the form $T=f(x, z)$, with two independent variables (Guiard, 2009). Using Fitts's (1954) classic data set as an illustration benchmark, this paper shows that a complete two-DoF approach considerably improves our understanding of Fitts' law data.

\footnotetext{
${ }^{1}$ Google Scholar (December 2, 2018) counts 7,748 citations of Fitts's (1954) article.
} 


\section{Fitts's (1954) Data and Fitts' Law}

Fitts's own formulation of the rule, which he gave explicitly in Fitts and Peterson (1964), reads

$$
T=a+b I D
$$

where $a$ and $b$ denote empirically adjustable coefficients. $T$ stands for mean movement time, and ID for what Fitts (1954) called the index of difficulty, which he defined as a function of target distance $D$ and target width $W$ :

$$
I D=\log _{2}\left(\frac{2 D}{W}\right)
$$

\subsection{The Evolution of Fitts's Claim and His Experimental Evidence}

The thesis submitted by Fitts in his 1954 article (see also Fitts, 1953) was in fact stronger than Equation 1 . He actually proposed the generalization that the ratio ID/T-in his interpretation an estimate of information transmission rate in bits/s-was constant, which implied the proportionality of movement time to $I D:^{2}$

$$
\frac{I D}{T}=k
$$

However, Fitts refrained from writing his first thesis in explicit mathematical form and presented it as just tentative, using exceedingly prudent words. For example he concluded the abstract of his 1954 paper as follows: "The consistency of these results supports the basic thesis that the performance capacity of the human motor system plus its associated visual and proprioceptive feedback mechanisms, when measured in information units, is relatively constant over a considerable range of task conditions. This thesis offers a plausible way of accounting for what otherwise appear to be conflicting data on the durations of different types of movements." (Fitts, 1954, p. 268, emphases added).

\footnotetext{
${ }^{2}$ There has been controversy on the necessity of a zero-intercept in Fitts' law (e.g., Soukoreff \& MacKenzie, 2004; Zhai, 2004). If possibly useful for comparison purposes, the intercept is uninterpretable in and of itself because the difficulty continuum has an arbitrary zero (Guiard \& Olafsdottir, 2011). For example Fitts's $I D=\log _{2}(2 D / W)$ zeroes out at the limit where $W$ becomes so large as to equal $2 D$-in no way the point where task difficulty zeroes out, but rather the point where the target interval begins to include the start point, meaning that the movement task disappears.
} 

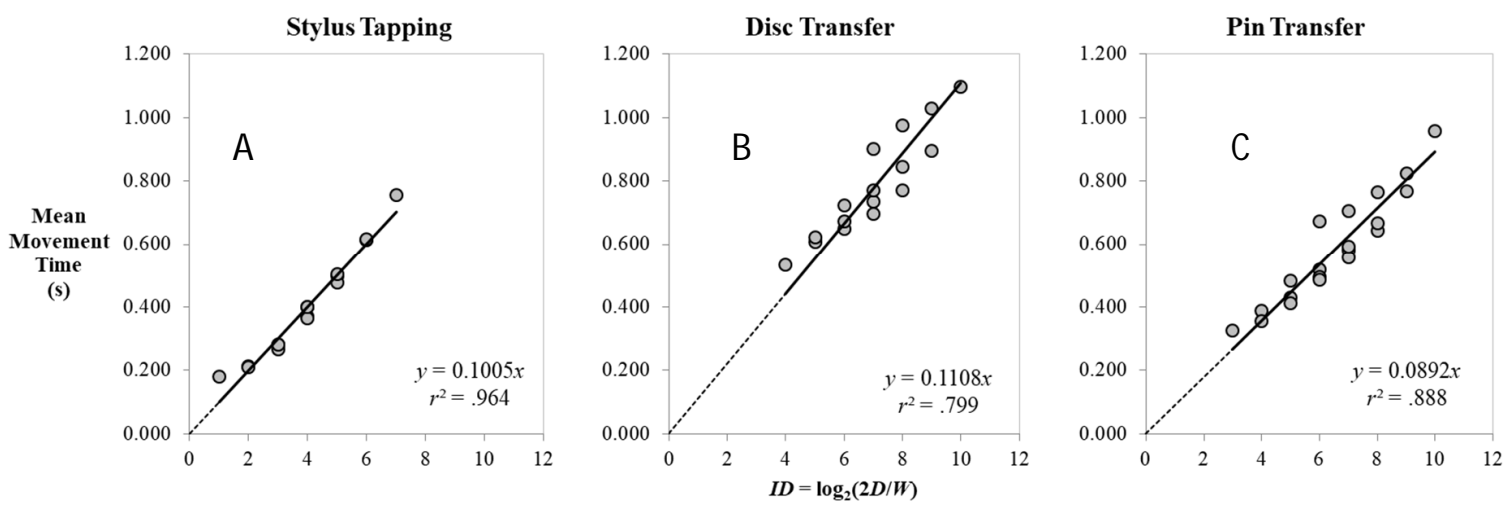

Figure 1. The movement time data of Fitts (1954, Tables 1, 2, and 3) with Equation 2 fitted.

Indeed Fitts's (1954) evidence came from three experiments on "different types of movement", namely, stylus tapping, disc transfer, and pin transfer. As is visible in Figure 1, which represents graphically the numerical data of his Tables 1, 2, and 3 (pp. 385, 386, and 388, respectively), the support for his proportionality claim was mediocre. Except for tapping the proportion of the variance of $T$ left unexplained by Equation 2 was rather high. ${ }^{3}$ Only the tapping experiment was an undisputable success.

In his next study, ten years later (Fitts \& Peterson, 1964), Fitts introduced two changes. For one thing he replaced his rather risky proportionality hypothesis with the more flexible twoparameter model of Equation 1, given it the explicit form of a mathematical formula (p. 104). The other change introduced by Fitts and Peterson is that the disc-transfer and the pin-transfer tasks of the 1954 study disappeared discreetly from the scene, leaving tapping as the exclusive focus. The fresh results that Fitts and Peterson (1964) obtained in a novel, discrete variant of the tapping task allowed them to conclude that "the times for discrete movements follow the same type of law as was found earlier to hold for serial responses" (p. 103). But here the meaning of the term "movement" is more narrow: Fitts was no longer referring to the variety of movements he had actually investigated in 1954 , but specifically to stylus-tapping.

That posterity usually gives credit to Fitts (1954) for the formulation of Equation 1 -which he in fact proposed ten years later with Peterson-is just a minor historic inaccuracy. What is more of a concern is that since Fitts and Peterson (1964) it has become a tradition to put

\footnotetext{
${ }^{3}$ With the intercept treated as a second free parameter, to match Equation 1a, the improvement is very small for the disc-transfer data $\left(r^{2}=.844\right.$ instead of .799$)$ and for the pin-transfer data $\left(r^{2}=.890\right.$ instead of .888$)$.
} 
aside two of the three carefully-run experiments that Fitts reported in full detail in his celebrated article of 1954.

\subsection{Comparative Merits and Weaknesses of Fitts's (1954) Three Experiments}

Fitts's (1954) three experiments are variations on the same theme of aimed movement. All three capitalized on the measurement of minimized $T$, all three involved the experimental manipulation of $D$ and $W$ using the same orthogonal design ${ }^{4}$, and all three used the same serial, or reciprocal protocol.

One weakness common to all three experiments is that they used the reciprocal protocol. Although that protocol has the obvious merit of allowing high rates of data production - which makes it practical in applied-research contexts-it has some drawbacks for the analytic mind, as later recognized by Fitts himself (Fitts \& Peterson, 1964; see also Guiard, 1997). As far as tapping is concerned, one drawback is that endpoint variability results in part from the execution of the movement and in part from the variability of the start point, inherited from the previous movement. Another drawback is that $T$ measures not just the time it takes to execute the current movement, but also the time it takes to correct the error inherited from the previous movement as well as the time it takes to prepare the next. Concerning the other two tasks, one drawback of the reciprocal protocol is to permit just the global indiscriminate timing of a cycle of different acts (grasping one object, transporting it, inserting it, moving the hand back, grasping the next object, etc.).

In one important technical regard the tasks of Exp. 2 and 3 were better suited to the study of aimed movement than tapping. The disc-transfer and the pin-transfer tasks demand that objects be put in place, thus forcing by construction a $0 \%$ error rate. This is a serious advantage over tapping, where a proportion of target misses inevitably occur, raising a serious concern about the reliability of the time measures. The speed and accuracy of performance always trading with each other in speeded tasks, if error is allowed both will tend to be affected by any manipulated factor. Indeed it is easy to check in Fitts's Table 1 (p. 385) that in his tapping experiment there was a

\footnotetext{
${ }^{4}$ In the two transfer tasks $W$ was simply the difference between hole diameter and pin diameter.
} 
high level of overall correlation between $T$ and error rate $(r=.82)$, clearly an undesirable outcome.

In the face of the tricky speed/accuracy trade-off problem Fitts (1954) took the traditional stance. His task instructions-specifically, "hit as many targets as you can" [in the 30s allotted to the trial] and "emphasize accuracy rather than speed" (p. 384, emphasis in the original)-were aimed at lowering the error rates down to a point where it may seem reasonable to ignore them. With less than $2 \%$ target misses on average in the tapping experiment, one may be tempted to conclude that Fitts's strategy was successful. Unfortunately, however, the intuition behind the traditional strategy is false. As has been known since the nineteen seventies, in general the form of trade-off functions is such that minute variation of the probability of error can be obtained at the expense of potentially considerable variations of speed. Worse-somewhat ironically-it is precisely at very low levels of error-rate that the variation of performance time can become enormous for extremely small differences in error percentages (Pachella, 1973; Wickelgren, 1977).$^{5}$

As far as mental chronometry, the target of the Pachella-Wickelgren objection, was concerned, the only serious response would have been to replace the measurement of response time with the study of entire speed/accuracy trade-off functions, that is, to accept to have to tackle two dependent measures at the same time. But such an option complicates things to a considerable extent and, unsurprisingly, the recommended revision never happened (Luce, 1986). Notice, however, that Fitts's paradigm has nothing to do with mental chronometry-it considers the chronometry of overt, rather than covert processes. If it is presumably impossible to obtain exactly $0 \%$ error in a mental chronometry experiment, it is in fact fairly easy to meet this requirement in an aimed-movement task: it suffices to make sure the process under study (here the movement) is complete at the time the timer is stopped, and this is precisely what Fitts (1954) did in his Exps. 2 and 3 with his disc- and pin-transfer tasks.

The weakness of Fitts's tapping paradigm with regard to the error issue has not escaped the attention of Fitts' law students. Following Crossman (1956), an abundant literature has accumulated on how to adjust the $T$ data in Fitts' law experiments so as to try to compensate for

\footnotetext{
${ }^{5}$ These authors were discussing reaction time specifically but their objection is quite general and obviously applies to the study of movement time.
} 
non-constant error rates. A practical consensus has been reached within some communities of researchers (Soukoreff \& MacKenzie, 2004; ISO, 2000) but the issue is still unsettled. Thus, all in all, it is the most fragile of Fitts's (1954) three experiments that posterity has retained. One reason for seriously considering his long forgotten Exps. 2 and 3 is that in them he elegantly avoided the complicated speed/accuracy trade-off problem that plagued his famous Exp. 1.

Thus with respect to the error issue Exps 2 and 3 were smarter than tapping. In another, more minor respect, however, they were not quite as smart. One astute option taken by Fitts in his tapping experiment was to use elongated rectangular target plates so that the tolerance constraint was manipulated selectively in the direction of the movement-giving $D$ and $W$ the status of strictly collinear lengths. In the disc-transfer and pin-transfer tasks, however, Fitts defined tolerance as a diameter difference, meaning that he manipulated $W$ both in the direction of the movement and in the perpendicular direction. Presumably a strictly $1 \mathrm{D}$ version of these tasks would have been preferable.

To this writer's knowledge the results of Fitts's (1954) disc-transfer and pin-transfer experiments have never been understood, not even by Kvalseth (1980), one of the few who attempted a complete reanalysis of Fitts's three tables. This author showed that all three scatter plots of Figure 1 are slightly more accurately summarized with a power function of the form $T=$ $a(D / W)^{b}$ with $b<1$, than they are with the logarithmic function of Equation 1. However, Kvalseth never explained why, using no more than two free parameters, the power model, which does an excellent job with the data of Figure 1A $\left(r^{2}=.986\right)$, does so mediocre a job with the data of Figure 1B $\left(r^{2}=.90\right)$ and $1 \mathrm{C}\left(r^{2}=.85\right)$. It is hard to be content with a mathematical summary that still overlooks up to $15 \%$ of the variance at hand.

In fact, so long as we stick to the traditional representations of Figure 1, there is no hope to do better than Kvalseth. As will be explained below, that representation is potentially misleading because it just shows a two-dimensional projection of a three-dimensional object. The portray given in Figure 1 of the data of Fitts (1954) - or for that matter of the data of any experiment using the Fitts time-minimization paradigm - is like the shadow of a vertical cylinder projected on a nearby wall: one dimension being lost, there is no warranty that one will be able to tell from that shadow whether the object is, say, a cylinder or a rectangular parallelepiped. 
Fitts's mistake-overlooking one of the two independent variables that his paradigm actually involves-has become routine in the field. To correct it we will have to make a detour through the polar/Cartesian analysis of Guiard (2009), to be summarized in the next section. The reward will be the realization that there is a great deal more structure in Fitts's data, and in the data from Fitts' law experiments in general, than is apparent in the traditional representation of Figure 1. We will discover that, contrary to a common belief, not just tapping but all three experiments of Fitts (1954) have delivered remarkably coherent and quite conclusive results. And this new complete visit to an old monument will be an opportunity to realize that Fitts' law is a more subtle matter conceptually than has been often thought.

\subsection{Double Checking the Explicandum: What is There to be Explained?}

Apparently confident in the received description of the published data, the literature on Fitts' law has been primarily concerned with the substantive-theoretical explanation issue. As he explained at length in an early writing (Fitts, 1953), Fitts's mind was inhabited by Shannon's (1948) then extraordinarily popular communication theory. Inspired by Hick's law of choice reaction time (Hick, 1952), he interpreted his findings as a further illustration, in the case of overt movement, of the limited information-transmission capacity of the human motor system.

Mainstream psychology, however, has always been reluctant to Fitts's attempt to apply Shannon's framework. Leaving Shannon aside, psychologists have preferred to explore other directions to explain Fitts' law. Wiener's (1948) cybernetics has been the main inspiration source

for the iterative correction theory of Crossman and Goodeve (1963/1983; see also Keele, 1968) as well as the stochastic optimized sub-movement theory of Meyer, Abrams, Kornblum, Wright, and Smith (1988). Plamondon and Alimi (1997) have proposed a kinematic theory based on the central limit theorem. Chaos theory has been conjured up (Flach, Guisinger, \& Robison, 1996), as well as nonlinear dynamical systems theory (Mottet \& Bootsma, 1999). Interestingly, the information-theoretic kind of explanation advocated by Fitts (1954) has been enjoying a revival, after three decades of pronounced discredit (Luce, 2003), with the emergence of human-computer interaction in the nineteen eighties (Card, English, and Burr, 1978; Gori, Rioul, \& Guiard, 2018; Hoffmann, 2013; MacKenzie, 1992; Soukoreff \& MacKenzie, 2004). 
The goodness of fit issue has also attracted considerable attention, especially in domains where Fitts' law serves application goals, such as ergonomics and human-computer interaction. Many minor amendments to the definition of the $I D$ of Equation $1 \mathrm{~b}$ have been put forth to improve the empirical fit of Fitts' law (see Plamondon \& Alimi, 1997, for a review). In many cases the logarithmic function was retained (e.g., Gori et al., 2018; Hoffman, 2013; MacKenzie, 1992, but other mathematical functions have been advocated for Fitts' law modeling, notably the power function (Kvalseth, 1980; Meyer et al., 1988).

This literature is essentially left aside in the present paper because it has in general taken for granted what we want to critically examine here, namely the description of the data of the Fitts paradigm. This paper is all about experimental facts and the methodology of establishing them. Rather than the explicans of Fitts' law (i.e., the substantive explanation issue), we focus on the explicandum: What does the quantitative empirical rule we call Fitts' law consist of? In fact we will see that the traditional factual description of Fitts' law, which he have inherited from Fitts himself (1954; Fitts \& Peterson, 1964), has a problem.

\section{How Many Independent Variables in the Fitts Paradigm?}

Behind Fitts' law, taken as an empirical fact, there is a hidden logical problem. The problem is somewhat troublesome but fortunately it has its solution.

\subsection{The Number of Degrees-of-Freedom Issue}

The time-minimization paradigm introduced by Fitts (1954) has acquired the status of a standard in Fitts' law research, both basic and applied. ${ }^{6}$ The idea is to take $T$ as the dependent measure and to manipulate target distance and target width independently, by means of an orthogonal $D \times W$ design. Letting $N$ denote the number of degrees of freedom (DoF) involved in this experimental manipulation, we have $N=2$.

\footnotetext{
${ }^{6}$ In principle the question tackled in the Fitts paradigm - the trade-off of speed and accuracy in the execution of aimed movement-is tractable using not just the popular time-minimization paradigm of Fitts (1954), but also the spread-minimization paradigm of Schmidt, Zelaznik, Hawkins, Frank, and Quinn (1979) and the dual-minimization paradigm of Guiard, Olafsdottir, and Perrault (2011).
} 
Notice that Fitts' law as formulated in Equation 1 involves just one independent variable, called $I D$. A 2D scatter plot is supposed to be sufficient to illustrate the law graphically, showing how $T$, represented on the vertical axis, is influenced by just one quantity represented on the horizontal axis. Letting $N^{\prime}$ denote the number of independent variables involved in the mathematical description of the law, we have $N^{\prime}=1$.

Thus the model supposed to answer the question asked in the paradigm has one less DoF than the question, a mismatch to be found in most mathematical descriptions of Fitts' law (e.g., Kvalseth, 1980; MacKenzie, 1992; Meyer et al, 1988). Virtually all models of the literature describe the outcome of the Fitts paradigm, which has two manipulation DoF, using a mathematical statement of the form $y=f(x)$, where $f$ represents a variety of functions, but where $x$ always stands for a single number, namely, the quotient of $D / W$.

Regardless of whether one is willing to adopt Fitts's own $I D$ or any other mathematical transform of the quotient of $D / W$, arguably much of the beauty of Fitts' law lies in the fact that it is so simple. Yet, even though apparently Fitts law theorists have not been alarmed by the mismatch that $N^{\prime}=1<N=2$, the disappearance of one manipulation DoF is an anomaly that requires an explanation.

Guiard (2009) called attention to the fact that the division of $D$ by $W$ involved in the calculation of any $I D$ is a binary operation, and that any binary operation involves a lossy compression of information. It is impossible to reconstruct the input elements, called the operands (two numbers), from the output, called the result (a single number). By definition, a binary operation compresses two DoF into one.

There is ambiguity in the usual mathematical notation of ratios such as that shown in Equation $1 \mathrm{~b}$, where two symbols, $D$ and $W$, serve to denote a single number-their quotient. The law can be written in two mathematically equivalent, yet conceptually different ways. The most popular of the two possible writings is that of Equation 1

$$
T=a+b I D
$$

with $I D=\log _{2}\left(\frac{2 D}{W}\right)$ 
which uses two successive mathematical clauses, a main clause and a subordinate clause. The main clause, of the form

$$
T=f(I D)
$$

where $f$ is a (usually linear) function, serves to define Fitts' law proper. The subordinate clause, of the form

$$
I D=g\left(\frac{D}{W}\right)
$$

where $g$ is another function, specifies how $I D$, the independent variable of the main clause, should be calculated from two quantities.

Now consider the other possible writing of Fitts' law, formed of a single mathematical statement in which the functions $f$ and $g$ are composed:

$$
T=f[g(D / W)]
$$

Equation 3a said quite explicitly that the law has just one independent variable-i.e., there is just one number on the right-hand side of the equation. As then explained in Equation $3 \mathrm{~b}$, that number is the result of some mathematical transform of the quotient of $D / \mathrm{W}$. In contrast, Equation 4 is ambiguous, the fractional expression $D / W$ on its right-hand side behaving pretty much like a Necker cube: that expression can be interpreted to denote either the quotient of the division of $D$ by $W$ (meaning $N^{\prime}=1$ ) or the two operands of that division (meaning $N^{\prime}=2$ ). What is unclear with Equation 4 is whether the division has been done (in which case one faces one number) or is to be done (in which case one faces two numbers, along with instructions for their use). However common in mathematical notation, such indetermination as to whether a 'ratio' like $D / W$ denotes the two inputs of the binary operation or its single output becomes problematic when it comes to the task of understanding how an experimental manipulation and the mathematical description of its results map onto each other.

As remarked by Guiard (2009), experimenters of all fields tend to follow the good sense rule that if the design of an experiment has $N$ manipulation factors, then the mathematical explication of the data from that experiment must have $N^{\prime}=N$ independent variables, simply because a hidden variable may play low-down tricks with them. In Section 3 below we will 
follow the $N^{\prime}=N$ rule scrupulously and, as we will see, new territories will open up. Fitts (1954) having used the same two-factor orthogonal design in his three experiments, ${ }^{7}$ in each case we will look for complete mathematical descriptions of the form $y=f(x, z)$, with two independent variables, rather than of the form $y=f(x)$, with a single independent variable as in the traditional understanding of Fitts' law. ${ }^{8}$

\subsection{Which Pair of Independent Variables? The Polar vs. Cartesian Answer}

What independent variables will occupy our two DoF? At first sight the Fitts paradigm involves three conceptually important quantities. These are (1) target distance $D$, which allows experimenters to take control over the amplitude of movement, (2) target tolerance $W$, supposed to allow experimenters to take control over the spread of movement endpoints, and (3) the quotient of the division of $D$ by $W$, to which Fitts (1954) was the first to call attention, and whose mathematical transformations have served to calculate a diversity of IDs. However, three independent variables is one too many because the paradigm has no more than two manipulation DoF.

In fact one faces a forced choice between two alternative description systems, which consider different, mutually incompatible DoF pairs, and that forced choice is similar to that between the Cartesian and the polar localization of a point $P$ in 2D space (Guiard, 2009). In 2D space, where the distinction is quite familiar, to use the Cartesian coordinates system means to specify the norms of the component vectors $\overrightarrow{\mathrm{Ox}}$ and $\overrightarrow{\mathrm{Oy}}$, and to use the polar coordinates system means to specify the direction and the norm of the resultant vector $\overrightarrow{\mathrm{OP}}$.

\footnotetext{
${ }^{7}$ For his tapping experiment Fitts used a three-factor design, crossing not just target distance $D$ and target tolerance $W$, but also stylus weight (1 lb. vs. $1 \mathrm{oz}$.), an auxiliary factor that turned out to exert virtually no effect on performance and to be involved in no interaction. Below we will leave aside stylus weight, the data being given on average over the two stylus weights.

${ }^{8}$ Below the contrast will be between incomplete one-DoF and complete $t w o$-DoF descriptions of Fitts' law data, but that count refers to the number of factors involved in the experiment and/or the number of independent variables shown on the right-hand side of mathematical equations. However, recall that $T$, by definition the dependent measure of the Fitts paradigm, constitutes a further independent dimension. Thus, taking $T$ into account, we will be contrasting incomplete two-dimensional descriptions of Fitts' law, of the form $y=f(x)$, versus complete threedimensional descriptions of the law, of the form $y=f(x, z)$.
} 
Polar
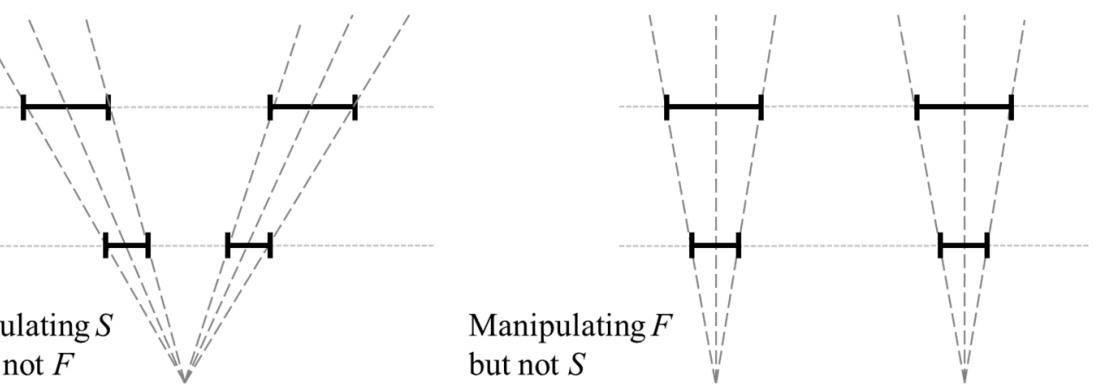

Manipulating $S$ but not $F$ but not $S$
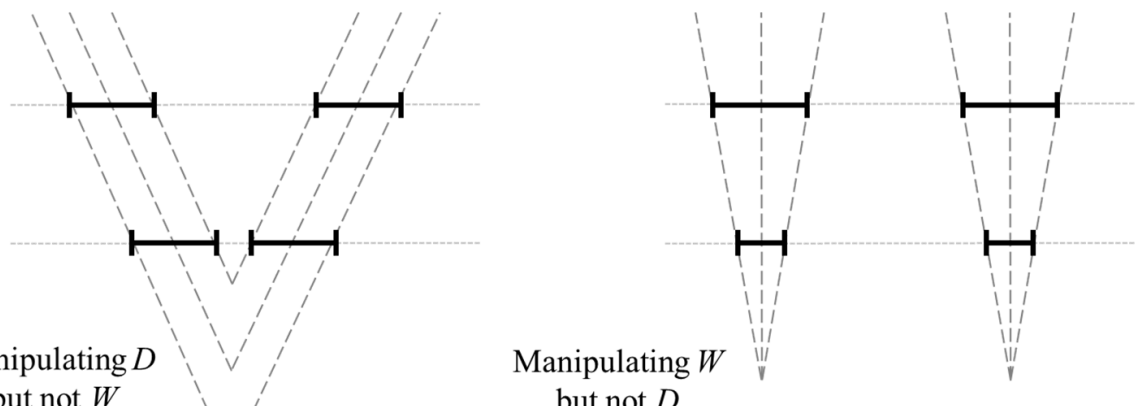

Manipulating $D$

but not $W$

but not $D$

Figure 2. The polar vs. Cartesian answers to the question of what it means to manipulate the two factors of the Fitts paradigm. The polar account (above) considers form $F$ and scale $S$, the Cartesian account (below) considers target distance $D$ and target width $W$. Each pair of horizontally-aligned thick line segments represents the two targets of Fitts's reciprocal protocol. This figure uses the space-scale diagram of Furnas and Bederson (1995), with space extending horizontally and scale vertically.

Of course the Fitts paradigm is one-dimensional, with $D$ and $W$ collinear, but the polar/Cartesian distinction still applies, as shown in Figure 2. The Cartesian description considers the two raw lengths $D$ and $W$, which jointly specify any task condition uniquely. The alternative polar description also specifies any task condition uniquely, but using a different pair of quantities: one is the quotient of $D / W$, a dimensionless measure that characterizes the form $(F)$ in 1D space of the movement task, and the other is some measure of scale $(S)$. Form being by definition a scale-independent attribute, it needs to be complemented with some scale measure, otherwise the task condition would not be specified uniquely.

Thus the polar vs. Cartesian distinction solves the paradox that while one DoF is missing in the usual plot of Fitts' law (Figure 1), one too many DoF seems to be conjured up in many 
discussions of the law. ${ }^{9}$ The explanation for the missing DOF is that the usual plot of Fitts' law offers an incomplete polar description of the data, one that considers $F$ but ignores $S$. The explanation for the DoF in excess in discussions of the law is the thoughtless hybridization of an incomplete polar description recognizing just $F$ with a complete Cartesian description recognizing both $D$ and $W$.

Figure 2 explains the logical relations borne by the four independent variables $F$ and $S$, and $D$ and $W$. The right-hand part of the figure explains the difference between the polar manipulation of $F$ (a global attribute of the target layout that can possibly be construed in terms of 'task difficulty') and the Cartesian manipulation of $W$ (a local attribute of the target). What differs is the independent variable that must be sacrificed. In the polar manipulations of $F$ (upperright panel) no independent definition of $W$ is available because the two available DoF are already occupied by quantities $F$ and $S$, and so the manipulated factor is really $F$, not $W$. In the Cartesian manipulation of $W$ (lower-right panel) no independent definition of the quotient of $D / W$ is available because the two DoF are already occupied by quantities $D$ and $W$, and so the manipulated factor is really $W$, not $F$.

Likewise, the left-hand side of Figure 2 explains the difference between the polar manipulation of $S$ and the Cartesian manipulation of $D$. Length $D$, which determines the average amplitude of the movement, will serve in the next section as our measure of $S$, meaning that $S$ and $D$ will refer to the same basic measure, yet one faces two different independent variables. Scale is a global attribute of the task: to manipulate $S$ (upper-left panel) is to manipulate all its measures, meaning that $W$ must be varied proportionally with $D$. In contrast, $D$ is a specific attribute of the target (specifying its distance from the start point), and to manipulate $D$ (lowerleft panel) demands that $W$ remain unchanged.

It is easy to see that Fitts (1954), who used in his three experiments the same design logic, crossing factors $D$ and $W$ orthogonally, initially conceived his paradigm in Cartesian terms. That was a perfectly sensible option, but for lack of a third DoF there remained no free slot for the quotient of $D / W$, a quantity of very special theoretical interest to him-to reiterate, that quotient

\footnotetext{
${ }^{9}$ Many Fitts' law students (e.g., Meyer et al., 1988; Sheridan, 1979) have expressed curiosity about the respective contributions of $D$ and $W$ to the effect of $I D$. In fact this is a logically intractable question because this triad of independent variables has only two DoF-once you know two of them, you known the third.
} 
cannot be defined independently of $D$ and $W$. There is no escape from the fact that the $D * W$ Cartesian description offers no DoF for the calculation of an $I D$, whether construed as a measure of information in Shannon bits or as a pure number (as, e.g., in Meyer et al., 1988).

There is one strength and one weakness in the methodology we have inherited from Fitts (1954) and which has become standard. The strength is an astute experimental paradigm based on the idea of time minimization, the weakness is the lack of control over the identity of the manipulated factors. In fact there are two problems. One problem is that while the Cartesian description serves to design the Fitts' law experiment, it is the alternative polar description that serves when it comes to the task of representing the data. This is quite problematic because a Cartesian experimental design cannot properly balance the effects of relevance to a polar analysis, meaning that Fitts's data, when tackled from the polar viewpoint, suffer from factor confounds - there is no guarantee that the main (average) effect of form $F$ will not be badly contaminated by an effect of scale $S$, and vice versa-for a detailed account of the possible artefacts, see Guiard (2019), and Gori, Rioul, Guiard, and Beaudouin-Lafon (2018).

The other problem is that the polar description system that has been used by Fitts' law students since Fitts (1954) is incomplete in the sense that it totally overlooks the DoF of scale.

\subsection{Scale: The Blind Spot of Fitts' law Research}

How is $T$ influenced by scale $S$ ? There is no room for such a question in the Cartesian view entertained by most predecessors of Fitts, along the lines of Woodworth (1899; see Elliott, Hensen, \& Shua, 2001). But the question arises immediately in the polar approach. Unfortunately, however, having adopted the polar understanding of his paradigm because the quotient of $D / W$ presents itself as the key quantity for an information theoretic approach, Fitts (1954) apparently did not pay attention to the other DoF and neither did his successors, so that the literature is essentially silent about the $T$ vs. $S$ relation.

Specifically, how should the dependent variable $T$ be affected by the manipulation shown in the upper-left panel of Figure 2, where $D$ and $W$ are varied proportionally so that task form, and by the same token task difficulty, remains constant? Not only is it likely that $T$ will be 
affected, the general shape of that dependency is easy to anticipate. Suppose that we rerun Fitts's tapping experiment at a fixed level of the quotient of $D / W$, say $F=32$ (meaning $I D=6$, using Equation $1 \mathrm{~b}$ ) but we rescale the task up and down over a large range, say from $S=1 \mathrm{~cm}$, a drastically miniaturized version of the movement task, up to $S=100 \mathrm{~cm}$, a drastically dilated version. The anatomy of the human perceptual-motor equipment being what it is, poor pointing performance will be obtained at both extremes of this scaling range. Movement times will be very long in the extremely miniaturized task condition, where $W=1 / 32^{\text {th }}$ of a cm is problematically small for the tip of a stylus, as well as in the extremely dilated condition, where $1 \mathrm{~m}$ is problematically large an amplitude for the arm of a seated human. Obviously the performance should be a great deal better in some intermediate region of the scale continuum.

These considerations essentially boil down to truisms, ${ }^{10}$ but they lead to non-trivial and testable predictions. Scale being a continuous quantity, we should expect the $T$ vs. scale function to be smooth. The big picture then must be a U-shaped relation-as indeed has been verified outside of the Fitts' law sphere in a diversity of aimed-movement experiments (e.g., Accot \& Zhai, 2001; Gibbs, 1962; Hess, 1973). ${ }^{11}$ We may further predict that any arbitrarily sampled segment of the $T$ vs. scale function must be decreasing or increasing if it happens to fall below or above the optimal region of the scale continuum, respectively. The function should be convex globally, but it can have linear portions. Therefore we must definitely predict a non-concave relation between $T$ and $S$.

Paying no attention to the scale dimension of their research problem, Fitts' law students have typically varied $S$ in an uncontrolled, inadvertent way. Suppose that by chance ${ }^{12}$ the performance measures have been taken in the intermediate, optimal region of the scale continuum and that the range of variation of $S$ is not too large. Because the $T$ vs. $S$ function may well be U-

\footnotetext{
${ }^{10}$ The bad reputation of truisms in science is undeserved. Indeed no merit is attached to their formulation because they consist of trivially true propositions. But truisms are true propositions and they have, in comparison with sophisticated or far-fetched propositions, the advantage of offering safe foundations for theory building, as recognized after all in axiomatic mathematics since Euclid.

${ }^{11}$ The general notion of a scale optimum is useful in a vast variety of contexts. In management, for example, Kuemmerle (1998) gathered evidence of an inverted U-shape relationship between the performance and the size of research and development laboratories.

${ }^{12}$ One simple reason why Fitts' law experimenters are in fact likely to go for about optimal ranges of $S$-even without conscious awareness of that variable-is that for any task a non-optimal scale level will induce an easily detectable effect of discomfort and fatigue.
} 
shaped, rather than V-shaped, it is quite possible to obtain locally a flat relation, neither increasing nor decreasing. Below such a case will be called isochrony.

\subsection{A Word on the Complexity Issue}

One central concern of this paper is the comparative complexity of Fitts's results, for each of his three experiments, with the data being described in (complete) polar vs. Cartesian terms. In general the complexity of an object may be thought of as the length of the shortest possible message that describes that object uniquely (e.g., Solomonoff, 1964 ). In the present context the descriptive messages we want to shorten are modeling equations and the unique objects whose complexity we want to evaluate are data sets-more specifically sets of $N$ triplets $\{x, z, y\}$, where $N$ denotes the number of different experimental conditions, where $x$ and $z$ specify a unique experimental condition, and where $y$ denotes the dependent measure.

Take the example of Fitts's tapping experiment. This experiment having explored 16 unique conditions, the data must consist of 16 triplets $=48$ numbers, it being understood that Fitts averaged the measures from all his participants. These 48 numbers can be arranged in a 16-line $\mathrm{x}$ 3-column array.

Fitts's Table 1 (p. 385) having six columns, obviously three of them are redundant, but which ones? The answer depends on the description system. From the Cartesian viewpoint the incompressible empirical information resides in columns C1-C3 of Fitts's table, columns C4-C6 being totally redundant. Indeed the $I D$ given in $\mathrm{C} 4$ is calculable from $D(\mathrm{C} 1)$ and $W(\mathrm{C} 2)$, the "index of performance" $I P=I D / T$ given in C5 is calculable jointly from $T(\mathrm{C} 3)$ and $I D(\mathrm{C} 4)$, and the performance rank given in C6 is calculable from $T$ (C3). In the Cartesian view the data thus consist of 16 triplets of the form $\{W, D, T\}$ reproduced in the right-hand part of Table 1 . 


\section{Table 1. Two minimal tabulations of Fitts's (1954) tapping data ${ }^{(*)}$}

\begin{tabular}{ccccccc} 
& Polar data & & & \multicolumn{3}{c}{ Cartesian data } \\
$\mathrm{F}(-)$ & $\mathrm{S}(\mathrm{cm})$ & $T(\mathrm{~s})$ & & $W(\mathrm{~cm})$ & $D(\mathrm{~cm})$ & $T(\mathrm{~s})$ \\
\hline 8 & 5.08 & 0.399 & & 0.635 & 5.08 & 0.399 \\
4 & 5.08 & 0.281 & & 1.270 & 5.08 & 0.281 \\
2 & 5.08 & 0.214 & & 2.540 & 5.08 & 0.214 \\
1 & 5.08 & 0.181 & & 5.080 & 5.08 & 0.181 \\
16 & 10.16 & 0.497 & & 0.635 & 10.16 & 0.497 \\
8 & 10.16 & 0.371 & & 1.270 & 10.16 & 0.371 \\
4 & 10.16 & 0.267 & & 2.540 & 10.16 & 0.267 \\
2 & 10.16 & 0.211 & & 5.080 & 10.16 & 0.211 \\
32 & 20.32 & 0.615 & & 0.635 & 20.32 & 0.615 \\
16 & 20.32 & 0.477 & & 1.270 & 20.32 & 0.477 \\
8 & 20.32 & 0.365 & & 2.540 & 20.32 & 0.365 \\
4 & 20.32 & 0.282 & & 5.080 & 20.32 & 0.282 \\
64 & 40.64 & 0.756 & & 0.635 & 40.64 & 0.756 \\
32 & 40.64 & 0.618 & & 1.270 & 40.64 & 0.618 \\
16 & 40.64 & 0.504 & & 2.540 & 40.64 & 0.504 \\
8 & 40.64 & 0.401 & & 5.080 & 40.64 & 0.401
\end{tabular}

(*) Note. $F$ is the quotient of $D / W$, and the measure of $S$ is given by $D$.

The alternative polar viewpoint dictates a different selection of columns. In the polar view all the empirical information from Fitts's tapping experiment is contained in columns C1, C3, and C4. The dependent measure $T$ is again found in column $\mathrm{C} 3$, but now column $\mathrm{C} 1$ specifies task scale $S$ and column $\mathrm{C} 4$ specifies task form $F$-the quotient of $D / W$. Thus, the data consist of 16 triplets of the form $\{F, S, T\}$, reproduced in the left-hand part of Table 1.

Thus each of Fitts's Tables 1-3, which contain the numerical data of his Exps 1-3, can be reduced to its minimum presentation, with no loss of information, in two different ways, meaning that the object we want to compress into a short mathematical formula exists in two different versions. Both the Cartesian $D * W$ description and to the polar $F * S$ description of Table 1 are complete, but there is no reason to expect the patterns of data to be identically easy to understand.

Let us turn from unique objects (the data) to their compressed descriptions (the modeling equations). Information compression is obviously an important goal of data modeling. When 
modeling a pattern of data we want to find a mathematical statement that summarizes the pattern as compactly as possible and with as little loss of empirical information as possible. In general it seems intuitively true that, as assumed in complexity theory, the more compact the mathematical summary, the simpler the data.

The smaller the number of free parameters in a good fit, the simpler the data (Akaike, 1973). This seems quite true under the assumption that all other things are equal, but we face the problem that modeling equations are compound objects composed of qualitatively different components such as variables, functions, operations, and adjustable parameters. Not to mention the problem that for some mysterious reasons some functions look obviously simpler, or more beautiful than others. For lack of a formal definition of data complexity we will follow the intuitive rule of thumb that in general the more conservations in the data, the simpler. Two characteristics of data sets seem to be of special importance in this regard, linearity and additivity.

A linear dependency-either the strict proportionality $y=a x$ or the affine function $y=a+$ $b x$-is simple in the sense that the effect of the independent variable $x$ on the dependent variable $y$ (i.e., the slope $\mathrm{d} y / \mathrm{d} x$ ) is the same at all levels of $x$. A nonlinear dependency is simpler if it can be linearized by means of a logarithmic transformation of $x$ (exponential function), or $y$ (logarithmic function), or both (power function) than if it cannot.

Given one dependent measure $y$ and two independent variables $x$ and $z$, the simplest possible combination of effects is the additive combination $y=f(x)+g(z)$, where $f$ and $g$ are functions. Additivity means that the effect exerted on the dependent variable $y$ by each independent variable is the same regardless of the value taken by the other independent variable.

\subsection{Recapitulation}

To sum up, Fitts's (1954) original work raises both a consistency concern and a completeness concern. The consistency concern is that having used the Cartesian concepts of target distance and target tolerance to design his experiment, Fitts switched to the alternative, polar system to formulate his mathematical answer, the mismatch entailing factor confounds (Guiard, 2009; Gori 
et al., 2018). The completeness concern is that his polar answer ignored the scale factor and thus lacked one DoF. In fact ignorance of the obvious possibility of an effect of scale in Fitts' law experiments has survived through sixty years of research.

In the next section we capitalize on the above framework to build side by side two complete mathematical descriptions, one in the polar $F * S$ space and the other in the Cartesian $D$ * $W$ space, of the data of Fitts's (1954) three experiments. Remaining essentially agnostic with regard to the substantive theory, we will look for data patterns and will try to capture them in parsimonious mathematical formulas.

\section{Revisiting Fitts's (1954) Data}

This section revisits the movement time data of all three experiments tabulated by Fitts (1954) in his Tables 1-3. Each experiment is considered first in polar $F * S$ space and then in Cartesian $D *$ $W$ space. Each time our task is to summarize the results as accurately and parsimoniously as possible with a mathematical equation, using both DOF at hand. ${ }^{13}$

\subsection{The Tapping Data (Experiment 1)}

\subsubsection{Polar Description of the Tapping Data.}

Fitts (1954) reported the numerical data of his tapping experiment in his Table 1 (p.385), giving 16 values of mean $T$, one for each cell of his $4 \times 4 D * W$ design. ${ }^{14}$

The tapping data are represented in Figure 3. As first noticed by Kvalseth (1980), the 16 data points are slightly better modeled by a power function than they are by the log function that

\footnotetext{
${ }^{13}$ In the search for the best possible description our default technique here is nonlinear regression, a robust technique that obviously accommodates the special case of linearity. Most of the best fits to be reported below were obtained from the online site http://www.xuru.org/rt/NLR.asp, which evaluates more than 100 mathematically "interesting" functions and ranks them according to goodness of fit.

${ }^{14}$ Fitts duly reported the 16 corresponding values of error rate. That other dependent variable, although reasonably low on average $(1.8 \%)$, correlates with $T(r=.82)$, casting a doubt on the validity of $T$ (Crossman, 1956; Soukoreff $\&$ MacKenzie, 2004). However, to take error rates into account-e.g., by trying to adjust the $T$ - would take us too far off the focus of this paper and so we will leave them aside, as Fitts (1954) did himself.
} 
Fitts had information-theoretic reasons to prefer. With all 16 data points pooled-that is, with the factor $S$ ignored-the best fit is

$$
T=0.171 F^{0.371}
$$

with $r^{2}=.988$. This equation can be rewritten as

$$
\log T=-1.764+0.371 \log F
$$

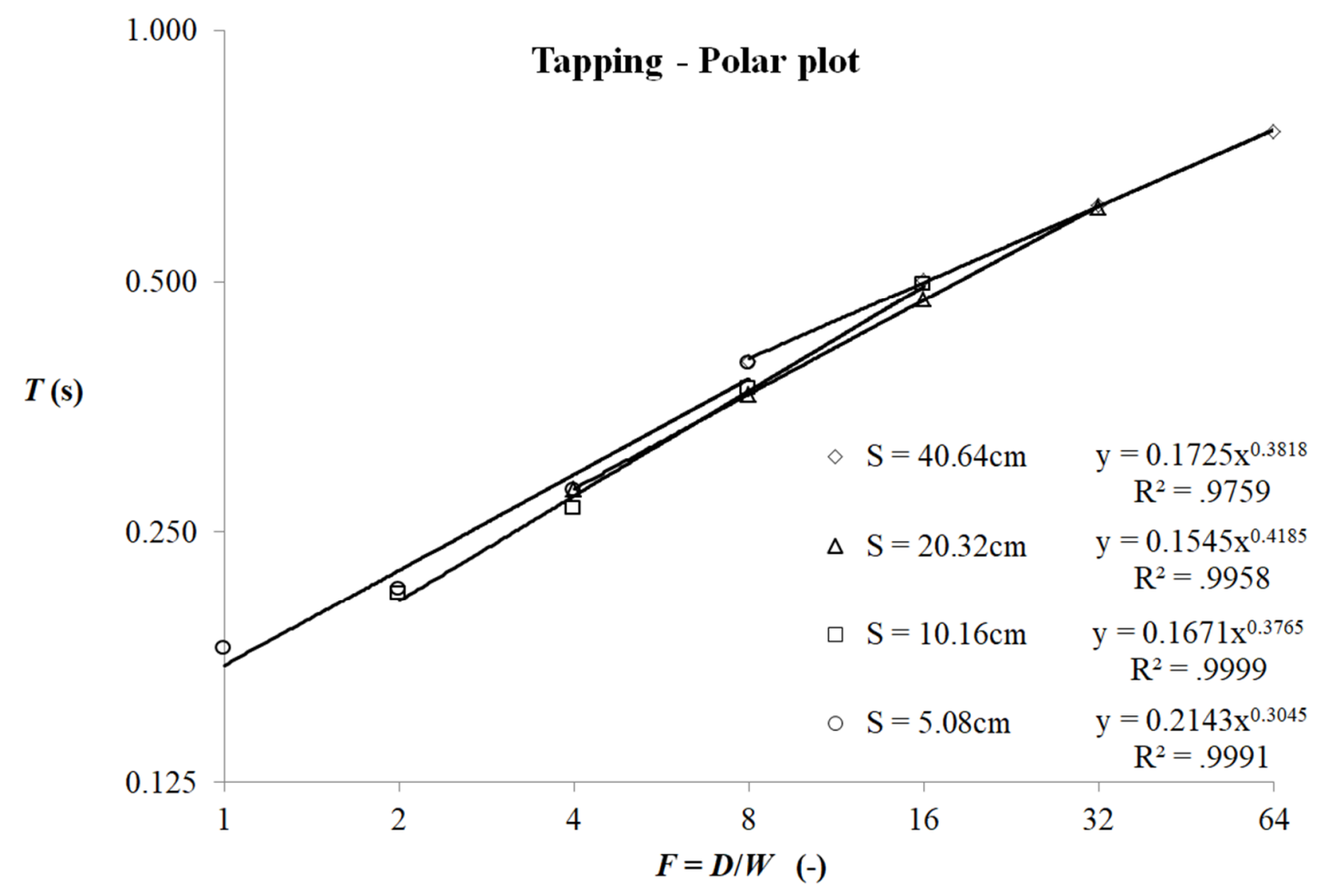

Figure 3. Complete polar $F * S$ representation of the tapping data. The plot is in $\log$-log coordinates.

However, there is something that Kvalseth (1980) could not see in his one-DoF reexamination of Fitts's data. It is not just the global scatter plot of $T$ vs. $F$, with all 16 data points pooled, that the power function models well-the power function does also very well at separate levels of scale, accurately modeling each of the four sets of four data point (see Table 2). 
Compared with the logarithmic model, the power model improves both the global fit $\left(r^{2}=.989\right.$ vs. $r^{2}=.964$ ) and the separate fits (on average $r^{2}=.993$ vs. $r^{2}=.973$ ).

Table 2. Global and separate fits of the logarithmic and the power models to the tapping data described in polar $F * S$ space.

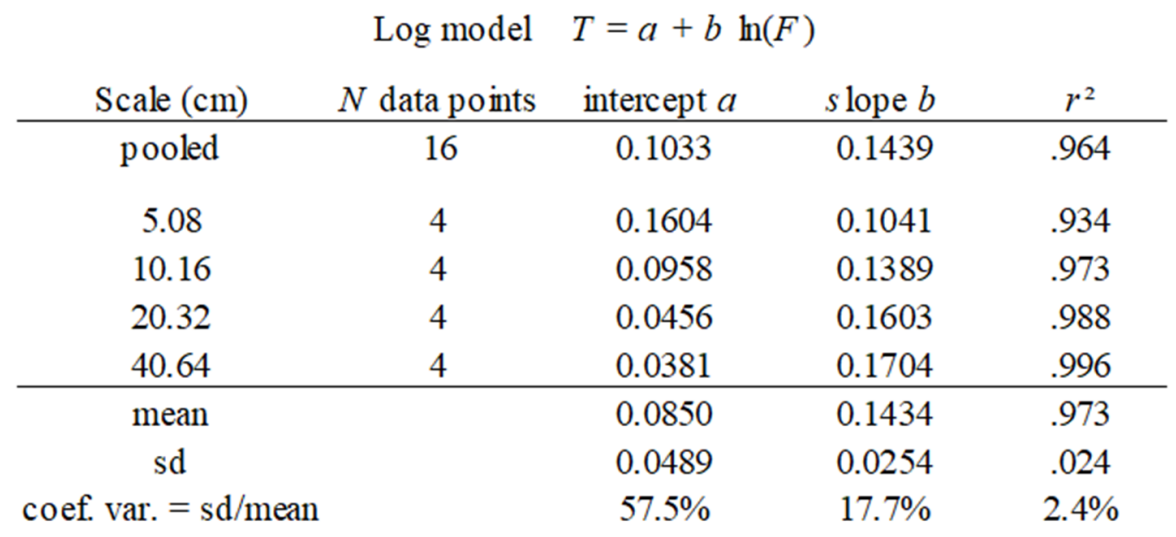

Power model $\quad T=a F^{b}$

\begin{tabular}{ccccc} 
Scale $(\mathrm{cm})$ & $N$ data points & scale coeff. $a$ & exponent $b$ & $r^{2}$ \\
\hline pooled & 16 & 0.1790 & 0.3544 & .989 \\
5.08 & 4 & 0.1725 & 0.3818 & .976 \\
10.16 & 4 & 0.1545 & 0.4185 & .996 \\
20.32 & 4 & 0.1671 & 0.3765 & .9999 \\
40.64 & 4 & 0.2143 & 0.3045 & .999 \\
\hline mean & & 0.1771 & 0.3703 & .993 \\
sd & & 0.0224 & 0.0413 & .010 \\
coef. var. = sd/mean & & $12.7 \%$ & $11.2 \%$ & $1.0 \%$
\end{tabular}

It also turns out that the estimated parameters of the $T$ vs. $F$ relation are better conserved across scale levels with the power than log model. That the power model improves that conservation is expressed quantitatively in Table 1 by a smaller coefficient of variation for parameter $a(11.2 \%$ vs. $17.7 \%)$ and especially for parameter $b(12.7 \%$ vs. $57.5 \%)$, which in both models captures the $F * S$ interaction. 
While the quasi-invariance of the exponent $b$ of Equation 5 (the slope in log-log coordinates) reveals the quasi-absence of an $F * S$ interaction, the quasi-invariance of the parameter $a$ (the intercept in log-log coordinates) reveals the quasi-absence of any scale effect on $T$. The latter observation, given the rather wide range over which Fitts manipulated scale (5$40 \mathrm{~cm}$ ), is a surprise: such quasi-invariance of a time measure across substantial variations of scale deserves a name, and that name is isochrony. If isochrony has been noticed for example in drawing and handwriting (Viviani and Terzuolo, 1982), it seems to have entirely escaped the attention of Fitts' law students. Isochrony is in no way a trivial outcome. It is impressively there in Fitts's tapping data (and in many data sets of the literature), but it must be emphasized that this need not be the case-we will see below that there was no isochrony whatsoever in Fitts's other two experiments.

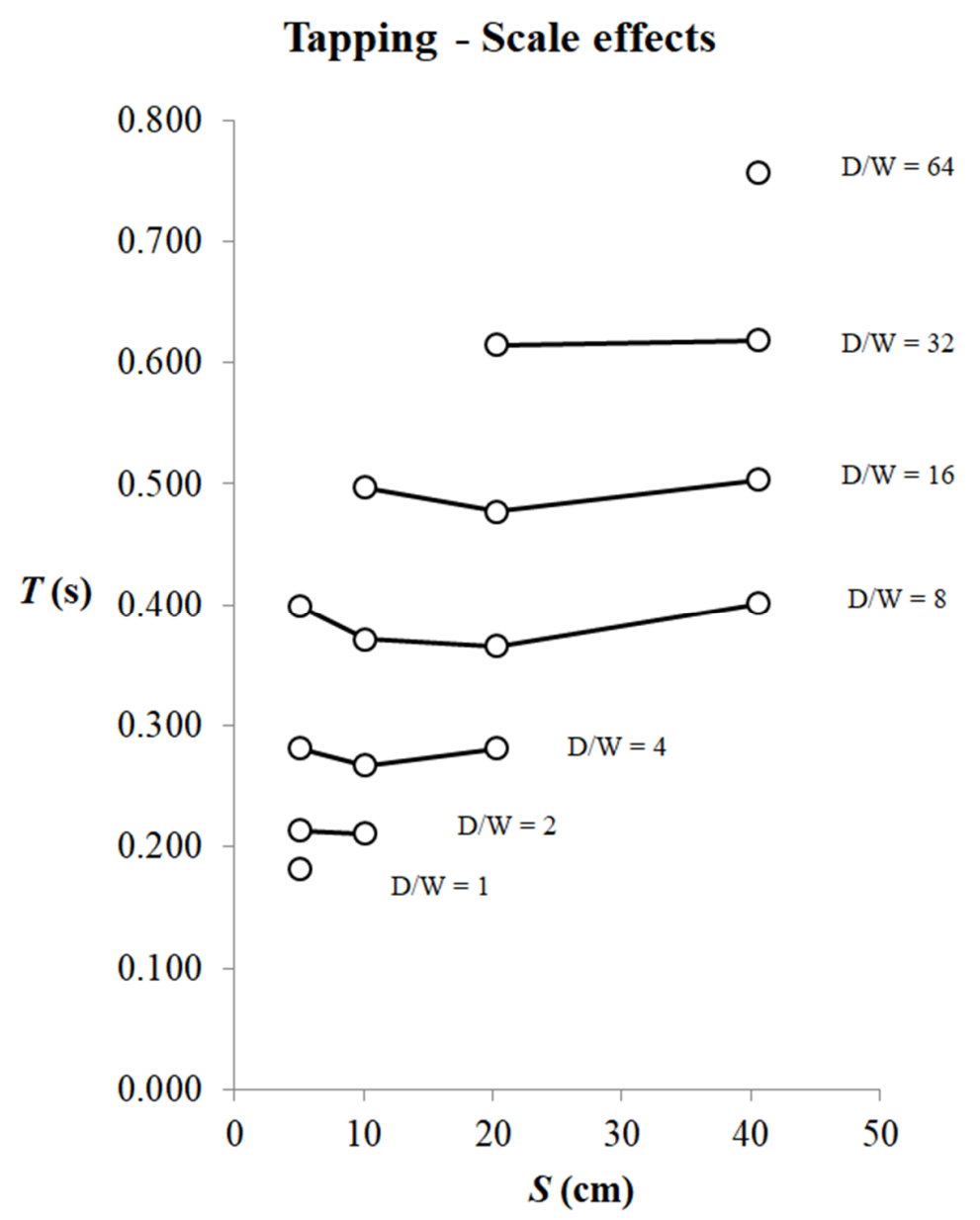

Figure 4. The other complete polar $F * S$ picture of Fitts's tapping data with $S$ and $F$ swapped to visualize the effect of scale. 
Figure 4 swaps the two independent variables to help visualize the effect of scale. $S$ is now plotted on the horizontal axis with $F$ appearing as a parameter. Notice that the effect of scale, however small, remains remarkably well structured. At all three levels of $F$ where three of more data points are available, thus allowing an evaluation of curvature, curve segments are convex, as expected. Indeed the slopes visible in the figure are rather shallow-were they steeper, the one-DoF model would not fit the data-but we do recognize fragments of U-shaped curves whose slopes all concur to suggest a minimum of $T$ in the region of $10-20 \mathrm{~cm}$. The left-hand side region of the figure exhibits three slightly negative slopes, suggesting that $5 \mathrm{~cm}$, the lowest scale level, was below the scale optimum for Fitts's tapping task. Symmetrically, on the right-hand side region of the figure we find three slightly positive slopes suggesting, no less consistently, that 40 $\mathrm{cm}$, the highest scale level for this experiment, fell above the scale optimum.

To recapitulate, the complete 2-DoF polar reexamination of Fitts's famous tapping data is teaching us three interesting lessons not available to the traditional 1-DoF approach. One is that Fitts was lucky in his tapping experiment because the effect of scale-an effect that had no room in his theoretical thinking-was so small in size as to be negligible. Second, he was lucky again because the level of interaction between $F$ and $S$ was negligible, the variations of scale perturbing to a surprisingly small extent the way the quotient of $D / W$, the determiner of his $I D$, influenced $T$. Hence the possibility for Fitts to model the $T$ data satisfactorily as a function of just one independent variable, $F$, and just two free parameters, as in Equation 1 or 5. And the third lesson is an explanation of the first two: the scale values that Fitts chose for his tapping experiment happened to fall in the optimal region of a U-shaped $T$ vs. $S$ function that happened to be remarkably flat.

Having noted that Equation 5 accounts for nearly $99 \%$ of the variance of $T$, it is not difficult to guess that the $1 \%$ loss of empirical information must have to do mainly with our decision to ignore the small, yet consistent scale effect described in Figure 4. This effect could certainly be modeled, but integrating this extra information in our formula so as to obtain a complete two-DoF polar model of tapping would offer a paltry accuracy gain. ${ }^{15}$

\footnotetext{
${ }^{15}$ Taking into account the minute (roughly linear) effect of $S$ on both the scaling coefficient $a$ and the exponent $b$ of the power function of Equation 5, a rather complicated two-DoF model obtains: $\log T=\log (0.15+0.0014 S)+$ $(0.4217-0.0027 S) \log F$, and the improvement of the fit is quite negligible, the $r^{2}$ progressing only from .988 to .992.
} 


\subsubsection{Cartesian Description of the Tapping Data}

Figure 5 shows the structure of the tapping data depicted in the alternative Cartesian $D * W$ space. The dependency of $T$ upon $W$ is essentially linear in log-log coordinates (with all four $r^{2}$ above .97), pointing to a power relation of the form

$$
T=a W^{b}
$$

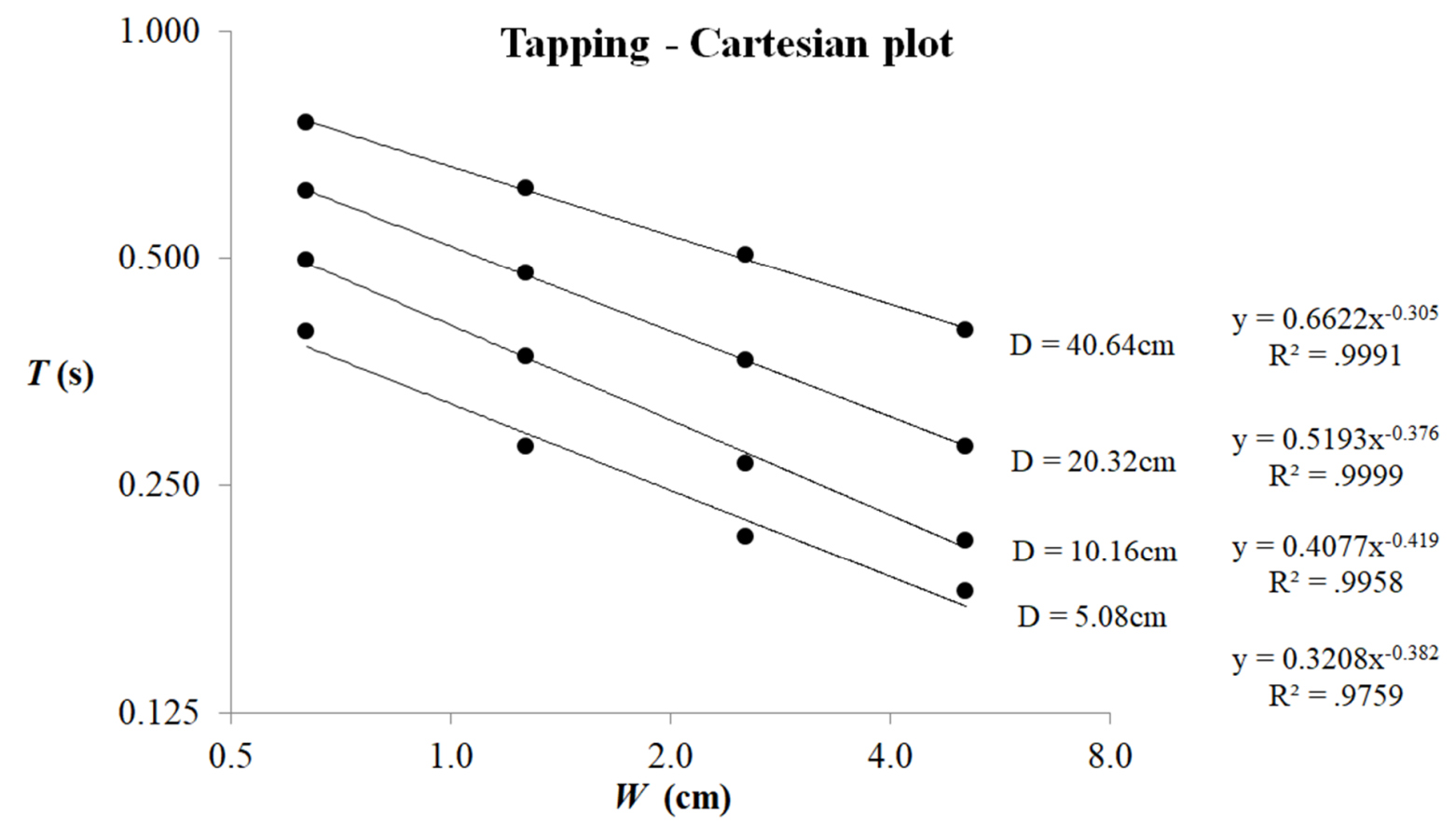

Figure 5. Complete Cartesian $D * W$ picture of Fitts's tapping data (log-log coordinates).

The four nearly parallel slopes can be summarized by simply averaging the four estimates of the exponent, obtaining $b=-0.37$. The next finding is that the scaling coefficient $a$ of the power model varies as a power function of $D$ :

$$
a=0.182 D^{0.349}
$$

with an impressive $r^{2}$ of .99999 . Thus, substituting for $a$ and $b$ in Equation 7, we obtain

$$
T=0.182 \frac{D^{0.349}}{W^{0.37}},
$$

which can be rewritten as 
$\log T=-1.7+0.35 \log D-0.37 \log W$

This two-DoF Cartesian model again accounts for $98.8 \%$ of the variance contained in the 16 values of $T$ tabulated in Fitts's Table 1.

\subsubsection{Comparison of the Polar and Cartesian Accounts}

Equation 10 looks pretty much the same as Equation 6. Since obviously $\log (D / W)=\log D-\log$ $W$, one might be tempted to conclude that the polar and the Cartesian analyses have led to the same result. Not so. For one thing, the two equations are mutually irreducible, as they refer to mutually exclusive descriptions of the independent variables. Then, most importantly, Equation 10 is a complete Cartesian $D^{*} W$ description whereas Equation 6, which involves $F$ but not $S$, is an incomplete polar description. Notice finally that Equation 10, with three free parameters for two independent variables (i.e., an average of 11/2 parameters per independent variable) has less flexibility than Equation 6 (two free parameters for one independent variable).

The structure of Fitts's (1954) tapping data being such that their modeling is about equally efficiently in both description systems, which system is preferable is a question we will leave for the substantive theory.

\subsection{The Disc-Transfer Data (Experiment 2)}

\subsubsection{Polar Description of the Disc-Transfer Data}

With Figure 6, built from the data of Fitts's Table 2, the benefit of a complete two-DoF polar analysis is particularly obvious. Now that the different levels of scale are properly separated, it is easy to see that in the disc-transfer experiment, unlike the tapping experiment, $S$ exerted a systematic effect on $T$. 


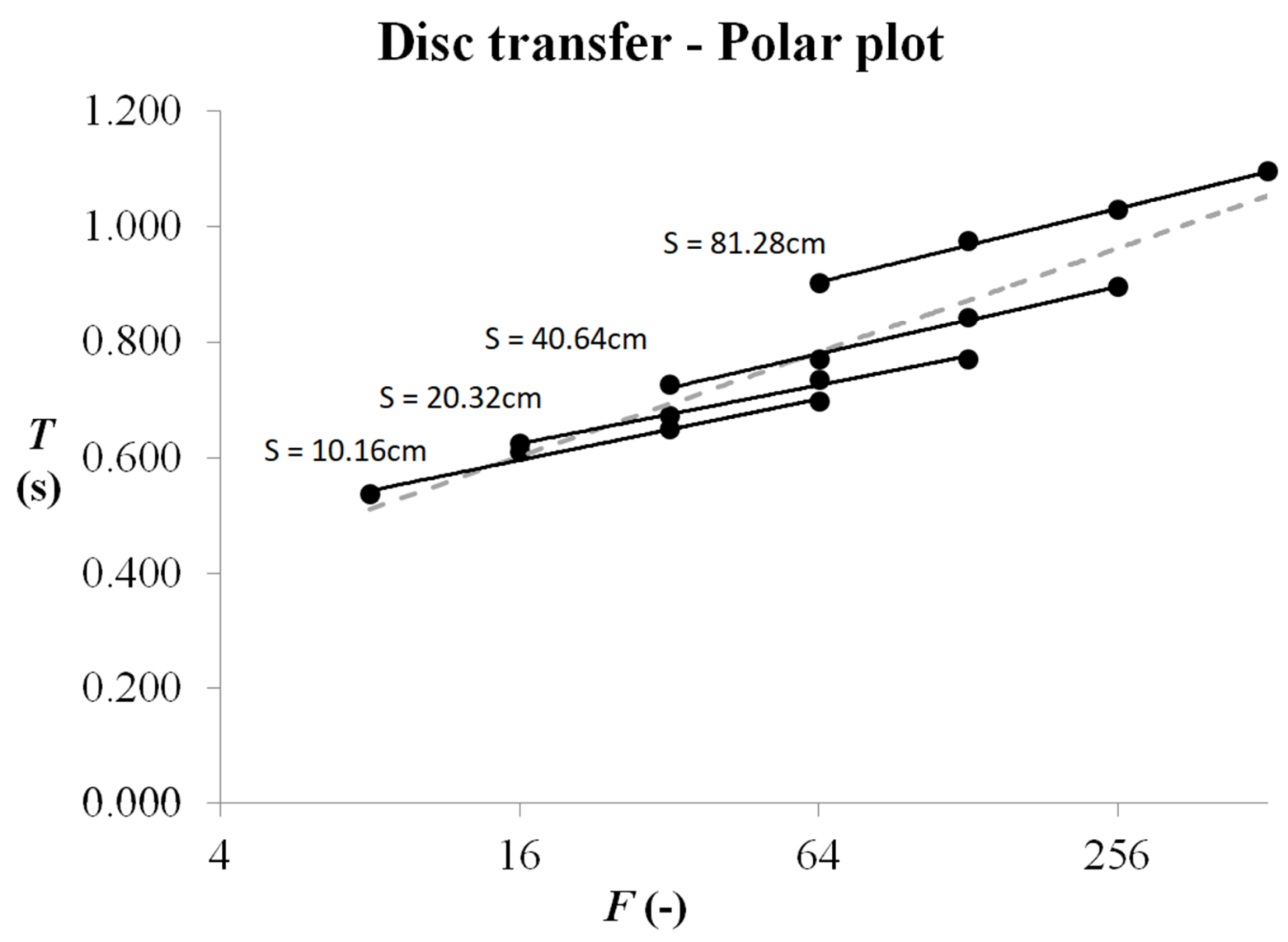

Figure 6. Complete polar $F * S$ picture of Fitts's disc-transfer data, to be compared with Figure 1B. Notice that the horizontal axis is logarithmic. The dashed line represents Equation 11 computed indiscriminately on all 16 data points. The continuous lines represent separate fits.

By the same token we understand the unpalatability of the disc-transfer data to Fitts and his successors, who have traditionally plotted the data as in Figure 1B. The effect of $S$ being unidentified, it looked like the $T$ vs. $F$ relation (i.e., Fitts' law) was polluted by random noise. That impression, however, is false. In no way were the results of Fitts's disc-transfer experiment more noisy than those of his tapping experiment, as is quite clear in the two-DoF representation of Figure 6. In the tapping data Fitts obtained a strong effect of $F$ combined additively with a very weak effect of $S$; in the disc-transfer data he still have had an additive combination but now we can see that the effects were both very strong. 
Table 2. Fit of the logarithmic model for the disc-transfer data represented in polar $F * S$ space

$$
\text { Log model } M T=a+b \ln (F)
$$

\begin{tabular}{ccccc} 
Scale $(\mathrm{cm})$ & $N$ data points & intercept $a$ & slope $b$ & $r^{2}$ \\
\hline pooled & 16 & 0.2403 & 0.1304 & .844 \\
& & & & \\
10.16 & 4 & 0.3844 & 0.0762 & .985 \\
20.32 & 4 & 0.4217 & 0.0730 & .992 \\
40.64 & 4 & 0.4259 & 0.0850 & .993 \\
81.28 & 4 & 0.5240 & 0.0916 & .997 \\
\hline mean & & 0.4390 & 0.0814 & .992 \\
sd & & 0.0517 & 0.0073 & .004 \\
coef. var. = sd/mean & & $11.8 \%$ & $9.0 \%$ & $0.4 \%$
\end{tabular}

The best description of the $T$ vs. $F$ relation that emerges from the nonlinear regression search is the logarithmic model $T=a+b \log F$. The fit is invariably excellent (on average $r^{2}=$ .992) if computed at separate scale levels (see Table 2). The pattern can be summarized with a simple model of the form

$$
T=a+b \log F
$$

where $b=0.081$ is the average slope of the linear function and where the intercept $a$ depends on $S$. As visible in Figure 6, and as can be checked from the values given in Table 2, the intercept $a$ is an approximately linear function of $S$ :

$$
a=0.369+0.0018 S
$$

with $r^{2}=.944$. Finally, by substituting for $a$ in Equation 11, we obtain the very simple additive model

$$
T=0.369+0.081 \log F+0.002 S,
$$

which accounts overall for $98.3 \%$ of the variance of $T$. This model has three free parameters but two independent variables. With an average of $1 \frac{1}{2}$ parameters per independent variable, it is 
actually less flexible than most models of Fitts' law, which have two free parameters for just one independent variable.

It should be noticed incidentally that the slope of Fitts' law visible in Figure 6 is much less steep than the global slope obtained in the traditional one-DoF treatment of Figure 1B. For tapping, where the scale effect had a negligible size, failure to disentangle it (Figure 1A) was of little or no consequence. However, for the disc-transfer data, where the scale effect is quite substantial, the consequence of that failure is a $50 \%$ overestimation of the slope of Fitts' law, as noted by Guiard (2009).

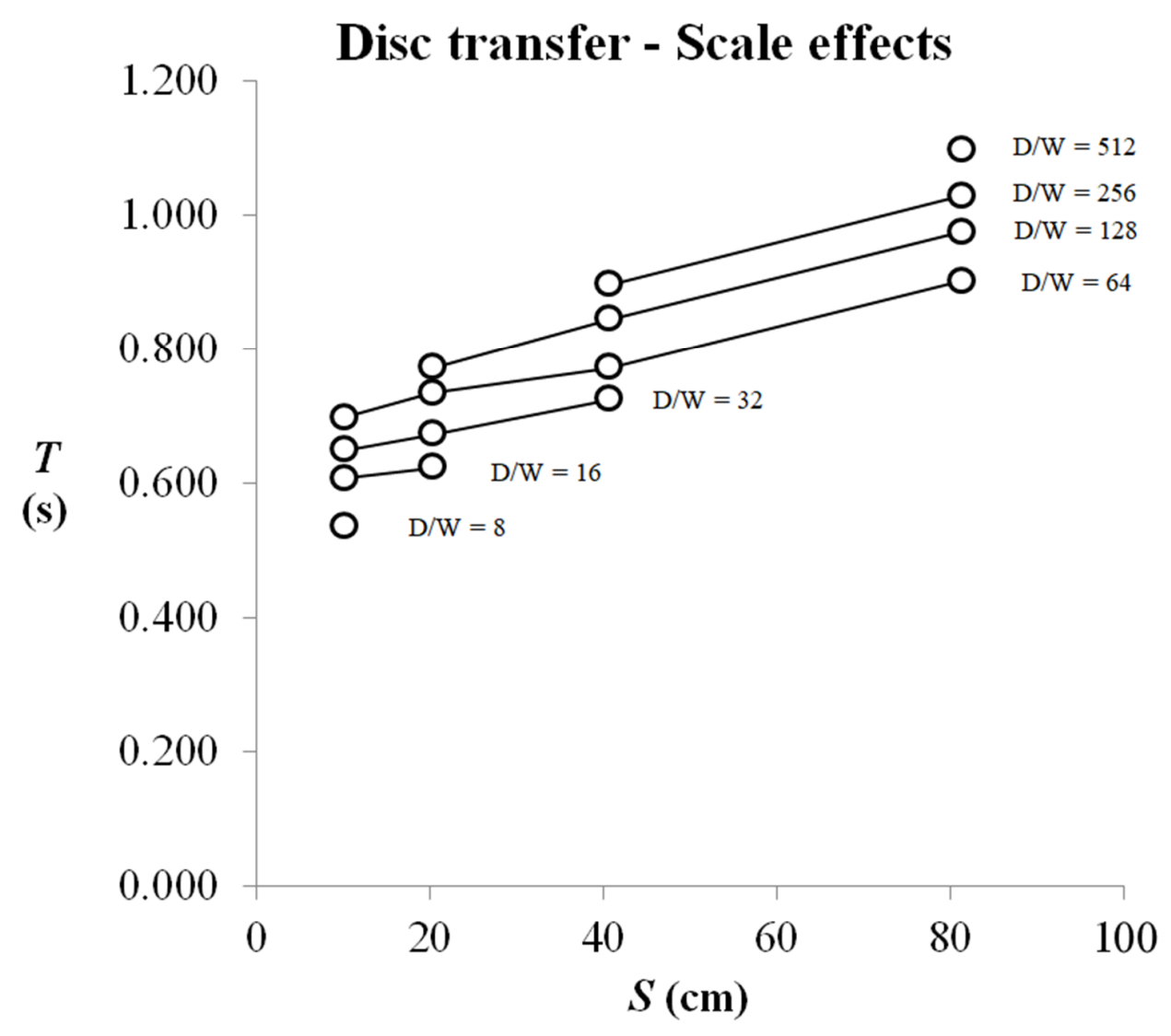

Figure 7. The effect of scale in the disc-transfer experiment, separately for each level of $F$.

The effect of scale is visualized in Figure 7. The pattern, though different from that of tapping, is again remarkably consistent. Here the more upscaling of the movement task, the worse 
the performance, meaning that the range of scale levels actually used in the disc-transfer task (10$80 \mathrm{~cm}$ ) fell in its entirety on the right-hand side of the U-shaped (or V-shaped) function. Unlike Figure 4, Figure 7 exhibits no convexity trends at the left-hand end of the curves, thus offering no clue as to the location of the scale optimum for the dis-transfer task-we just see that the optimum happened to fall somewhere below $10 \mathrm{~cm}$, a little below the scale optimum for tapping. In that particular experiment, and for that particular range of $S$ levels, the curve segments are neither convex nor concave, but roughly linear.

Having replaced the traditional one-DoF description of Fitts' law with the two-DoF model of Equation 13, we now realize that the law held just as well in Exp. 2 as it did in Exp. 1. While the tapping experiment revealed just Fitts' law, the disc-transfer experiment revealed Fitts' law plus a substantial effect of scale. The results of Exp. 2 help realize that something very special happened in Exp. 1: in tapping not only was Fitts able to demonstrate the simple law of variation which we now call Fitts' law, he also obtained, without noticing it, isochrony, a nearly perfect conservation of $T$ across pretty large variation of scale (Viviani \& Terzuolo, 1982). Whether or not isochrony should be considered a defining characteristic of Fitts' law is a question we postpone to the final discussion.

\subsubsection{Cartesian Description of the Disc-Transfer Data}

As shown in Figure 8A, $T$ varies linearly with $\log W$, and the effects of the two Cartesian variables are essentially additive, with $D$ affecting the intercept but not the slope of the $T$ vs. $\log$ $W$ function. We can describe the pattern as

$$
M T=a+b \log W
$$

where the slope can be estimated as the average of the four slopes, $b=-0.081$, and where the intercept $a$ varies as a function of $D$ (see Table 3). 
A

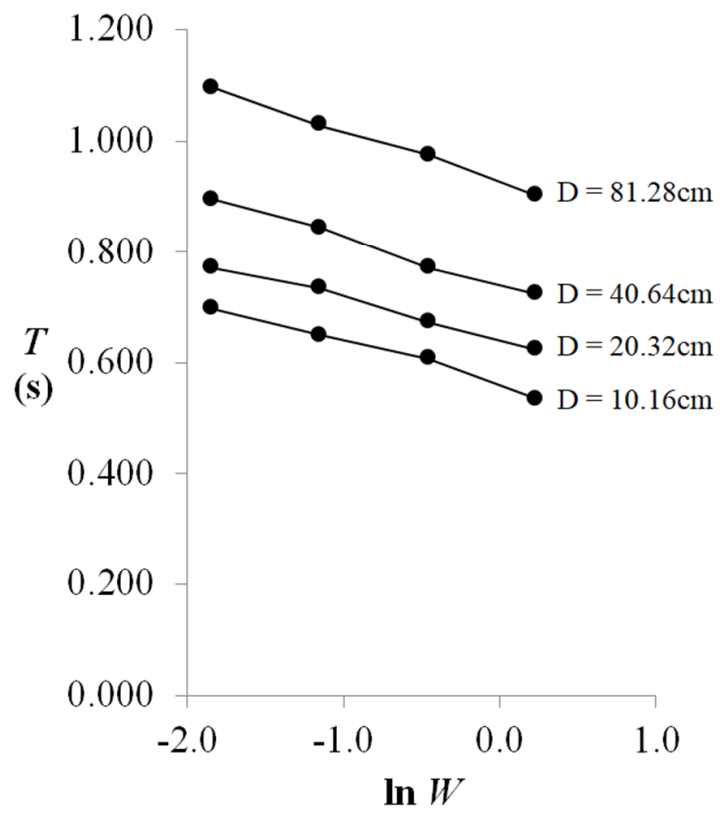

$\mathrm{B}$

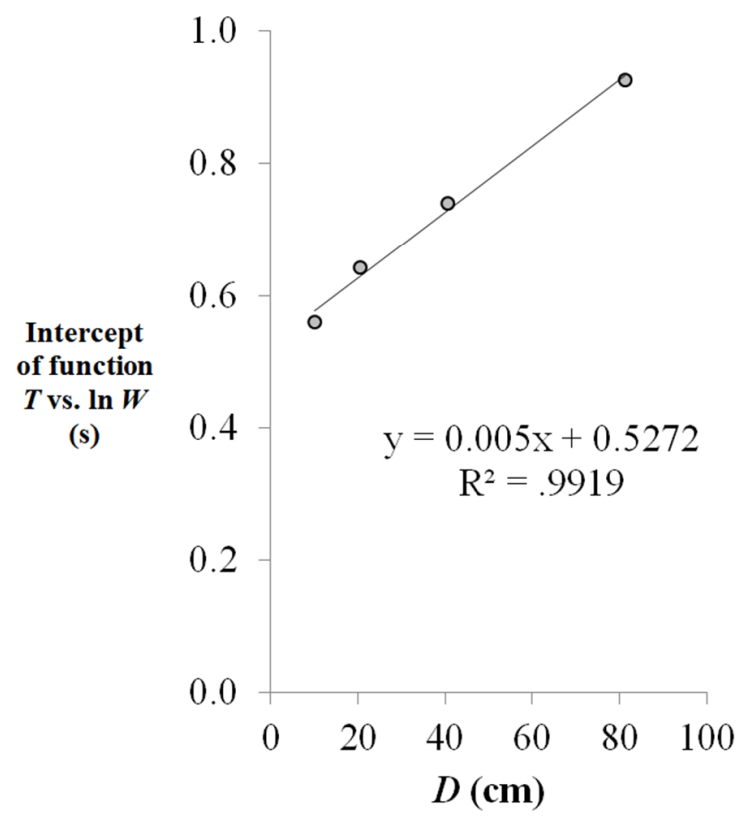

Figure 8. A: Complete Cartesian $D * W$ representation of Fitts's disc-transfer data. B: Effect of $D$ on the intercept of the $T$ vs. $\log W$ function.

That intercept turns out to vary about linearly with $D$ (Figure $8 \mathrm{~B}$ ), and so the data can again be modeled with an additive equation:

$$
T=0.527+0.005 D-0.081 \log W
$$

with at least as good a fit $\left(r^{2}=.991\right)$ as with the polar description $\left(r^{2}=.983\right)$. Thus, the two alternative two-DoF descriptions deliver similarly simple pictures of the data from Fitts's disctransfer experiment. In both cases a simple additive model summarizes the data very accurately. 
Table 3. Fit of the logarithmic model for the disc-transfer data represented in Cartesian $D * W$ space

Log model $T=a+b \log W$

\begin{tabular}{ccccc}
$D(\mathrm{~cm})$ & $N$ data points & intercept $a$ & slope $b$ & $r^{2}$ \\
\hline pooled & 16 & 0.7175 & -0.0814 & .164 \\
& & & & \\
10.16 & 4 & 0.5610 & -0.0762 & .985 \\
20.32 & 4 & 0.6415 & -0.0730 & .992 \\
40.64 & 4 & 0.7407 & -0.0850 & .993 \\
81.28 & 4 & 0.9269 & -0.0916 & .997 \\
\hline mean & & 0.7175 & -0.0814 & .992 \\
sd & & 0.1366 & 0.0073 & .004 \\
coef. var. $=$ sd/mean & & $19.0 \%$ & $9.0 \%$ & $0.4 \%$
\end{tabular}

\subsection{The Pin-Transfer Data (Experiment 3)}

It is not in his tapping experiment, now very famous, but in fact in his much less popular Exp. 3 on pin-transfer that Fitts actually invested the most effort. For this final experiment he recruited more participants (20, instead of 16 in Exps. 1 and 2), offered them "somewhat more practice" (p. 387 ), investigated more conditions (20 instead of 16), and ran more daily sessions per participant (three instead of two).

At first sight the pin-transfer task does not seem to differ much from the disc-transfer task. In both cases the task consists of inserting a protrusion into a hole, and the error rate is $0 \%$. Yet, as we will see, the results are strikingly different.

\subsubsection{Polar Description of the Pin-Transfer Data}

The polar picture, shown in Figure 9, is entirely different from that of the first two experiments. To begin with, consider the $T$ vs. $F$ relation separately at any given level of scale. The relation is 
still simpler than Fitts' law, being almost perfectly linear in double-linear coordinates (with $r^{2} \geq$ .99):

$$
T=a+b F
$$

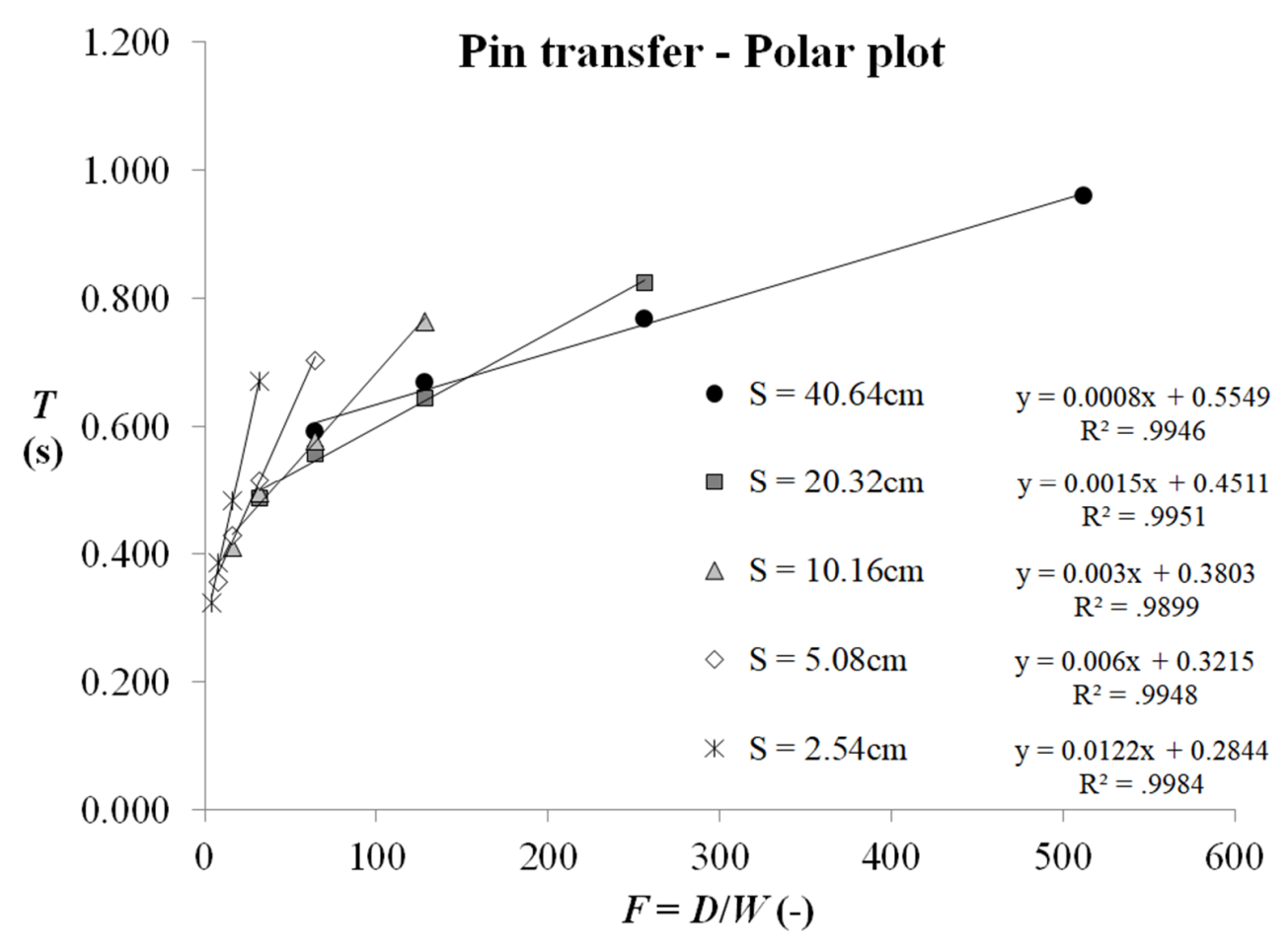

Figure 9. Complete polar $\boldsymbol{F} * S$ representation of the pin-transfer $\boldsymbol{T}$ data.

Such an accurate linear relation in Fitts's own data is yet another surprise. It should be emphasized that the remarkable linearity of Figure 9 was undetectable in the traditional one-DoF polar approach. Ignoring the five different levels of scale, and thus being confronted with twenty indiscriminate data points, one had the overwhelming_but quite wrong-impression of a concave relation between $T$ and $F$.

This, however, is just half of the story. The other discovery is a considerable amount of interaction between the two independent variables of the polar description, with both the intercept and the slope of the linear $T$ vs. $F$ relation being strongly dependent on scale. 

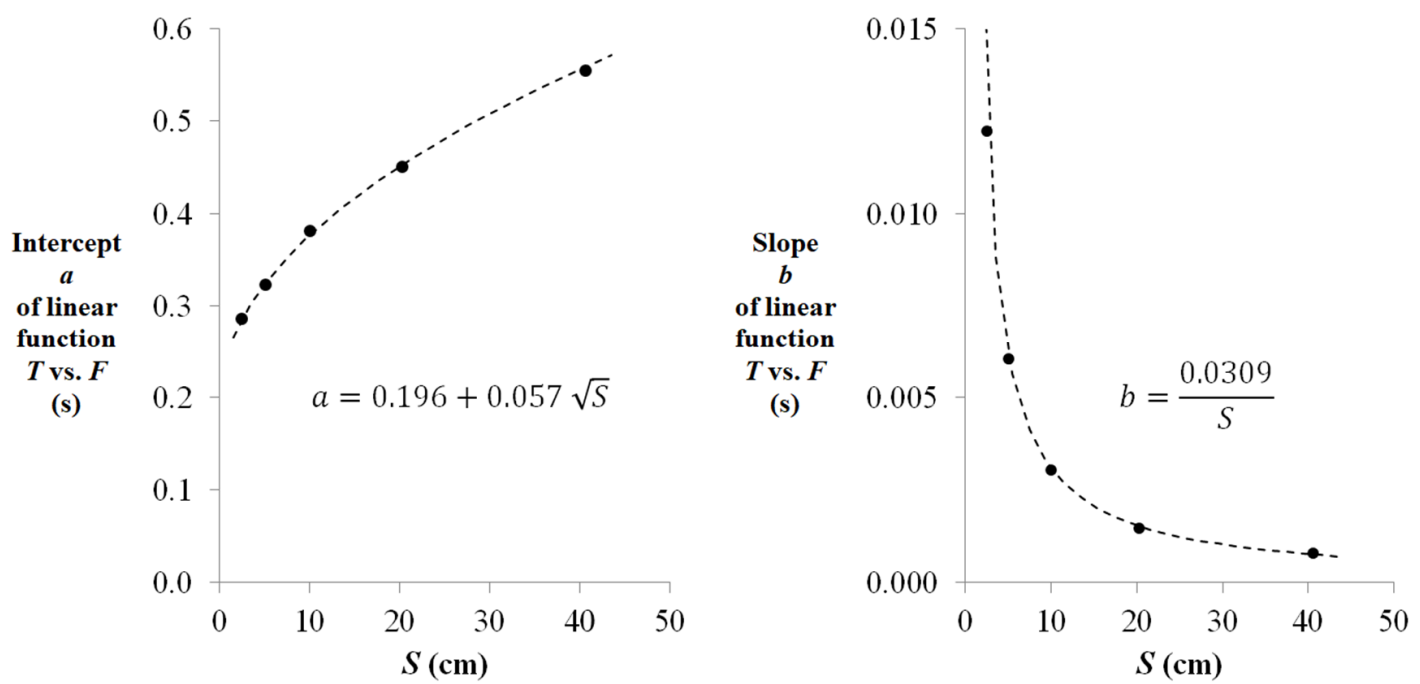

Figure 10. The $F * S$ interaction in the pin-transfer $T$ data.

As shown in Figure 10, these dependencies are both remarkably orderly. The intercept of Equation 15 is very well modeled as a square root function of $S$ (with $r^{2}=.999$ ), and the slope as proportional to $1 / S$ (again with $r^{2}=.999$ ). Numerically,

$$
\begin{aligned}
& a=0.196+0.057 \sqrt{S}, \\
& b=\frac{0.0309}{S} .
\end{aligned}
$$

Substituting for $a$ and $b$ in Equation 15, we obtain the complete two-DoF model

$$
T=0.196+0.031 \frac{F}{S}+0.057 \sqrt{S}
$$

which offers an impressively accurate summary of the 20 data points of Figure $9\left(r^{2}=995\right)$.

Equation 18 shows an addition of three terms, but notice that the second term combines $F$ and $S$ : the two polar variables interact very strongly with each other. In this sense the polar structure of the data is a great deal more complicated in Fitts's Exp. 3 than it was in the first two experiments. 


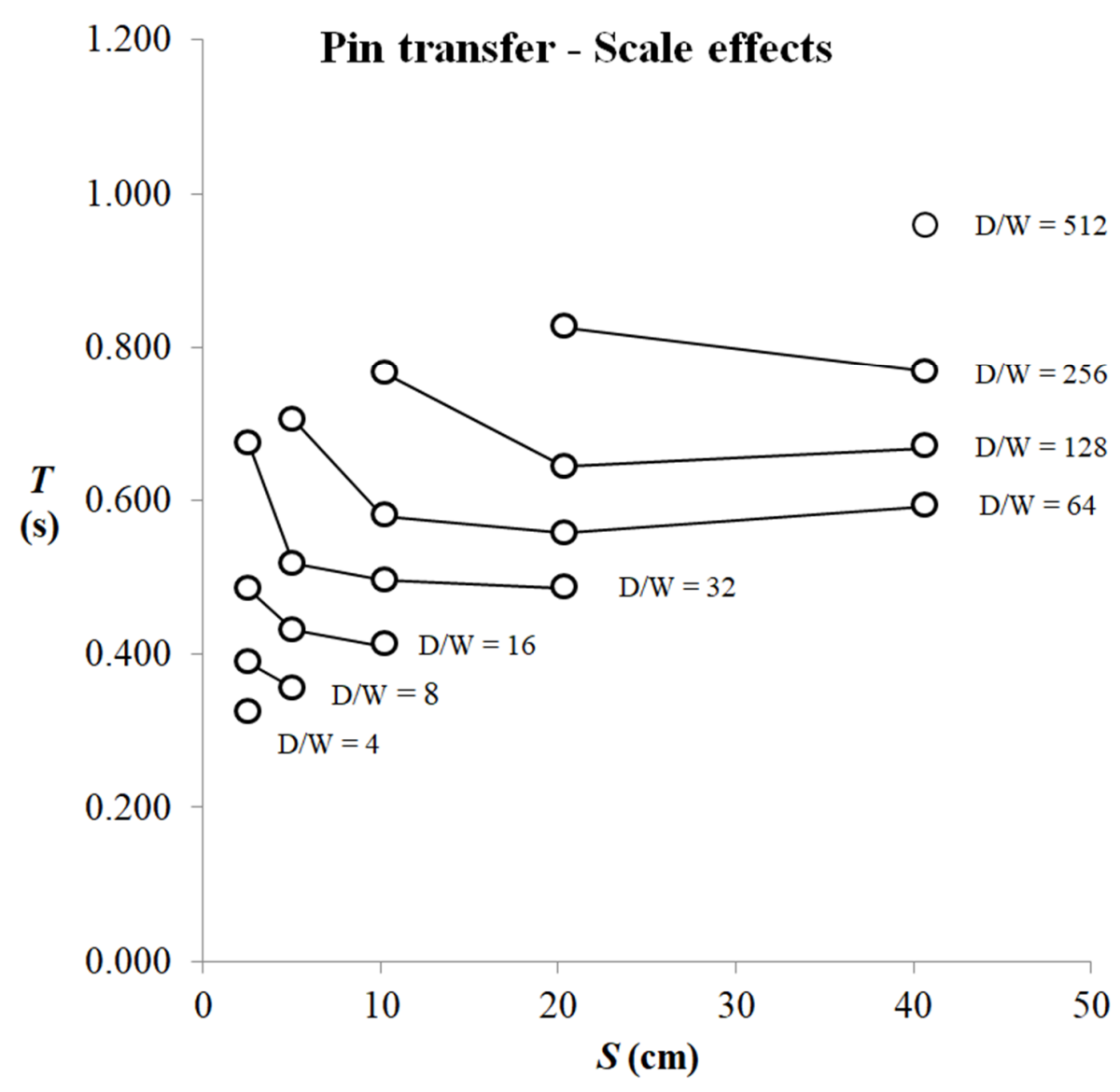

Figure 11. Movement time vs. $S$ at the eight different levels of $F$ of Exp. 3 .

This is not to say that in this third data set the effect of scale is complicated. Again we have a perfectly coherent pattern, visible in Figure 11. We can see that the curve segments are coherently convex, with slopes generally pointing to an optimum located at about $20 \mathrm{~cm}$, similar to that found in tapping. Of the 12 pairs of data points that contribute slope information in Figure 11 only one diverges, to a small extent, from the general trend. The set of slopes point most consistently to the presence of a scale optimum located at about $20 \mathrm{~cm}$, with particularly steep negative slopes below that optimum. 


\subsubsection{Cartesian Description of the Pin-Transfer Data}

Perhaps the most intriguing result of the present study is that obtained in the Cartesian $D * W$ analysis of the pin-transfer data. Having just found that the results of this final experiment are complex when described in $F * S$ space, now let us see how remarkably simple they become when described in the alternative $D * W$ space.

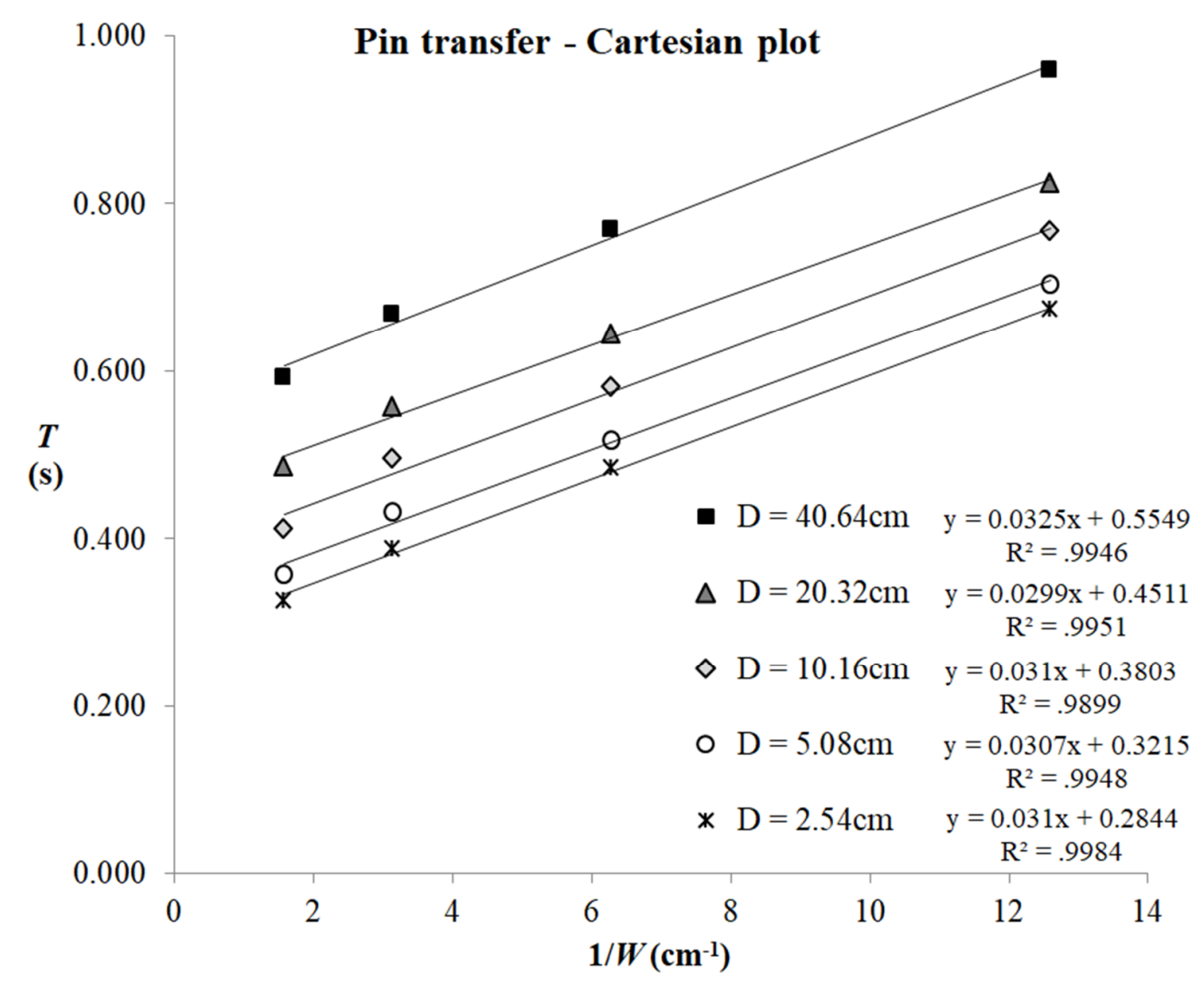

Figure 12. Complete Cartesian $D * W$ representation of Fitts's pin-transfer $T$ data.

At any given level of $D, T$ decreases nonlinearly with $W$. As visible in Figure 12, $T$ can be modeled as a linear function of the reciprocal of $W$ :

$$
T=a+b \frac{1}{W}
$$


with $r^{2} \geq .99$ for each of the five conditions of distance.

Figure 12 also shows that the two independent variables of the Cartesian description combine their effects on $T$ in a neat additive manner. While the intercept $a$ of Equation 19 is strongly dependent on $D$ (Figure 13A), the slope $b$ is remarkably constant across distance conditions (Figure 13B). The five estimates of $b$ range from a minimum of 0.03 to a maximum of 0.0325 , yielding a coefficient of variation of less than $3 \%$. Thus the mean value $b=0.031$ allows us to estimate the slope parameter of Equation 19 with virtually no loss of empirical information.
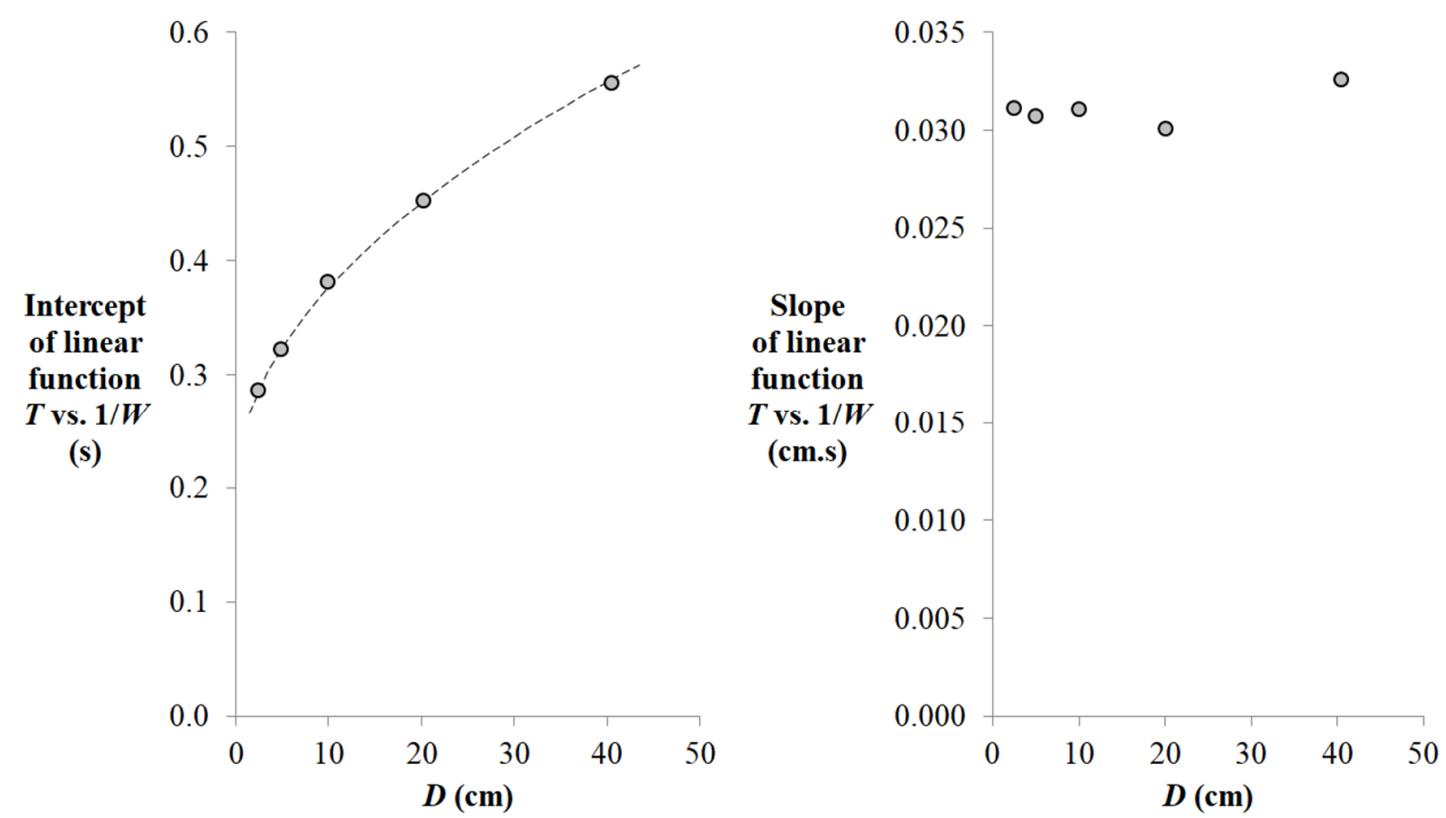

Figure 13. Effect of $D$ on the intercept but not the slope of the linear relation linking $T$ to $1 / W$. The dashed line represents Equation 20.

On the other hand, the dependency upon $D$ of the intercept of Equation 19 (Figure 13A) is very accurately modeled with the square root function

$$
a=0.196+0.057 \sqrt{D}
$$

with $r^{2}=.999$. 
It just remains to substitute for $a$ in Equation 19 to obtain a complete Cartesian description of the pin-transfer data:

$$
T=0.196+0.057 \sqrt{D}+\frac{0.031}{W}
$$

With an average of just $1 \frac{1}{2}$ free parameters per independent variable, this simple additive model accounts for $99.5 \%$ of the variance contained in the $20 T$ values of Fitts's Table 3 (p. 388).

\section{Discussion}

Of each of the three data sets tabulated by Fitts (1954) two complete alternative mathematical descriptions, one polar and the other Cartesian, have been elaborated in parallel. While the novelty of the above polar analysis is its completeness, $T$ being analyzed as functions of two (no less than two) independent variables, the novelty of the Cartesian analysis is its coherence: the quotient being carefully put aside, $T$ is analyzed as a function of two (no more than two) independent variables. New findings have emerged out of these old data, casting light on the essence of the empirical relation we call Fitts' law. We start by discussing the results of the polar analyses.

One result that could not possibly have been suspected in the traditional one-DoF polar approach is the presence of remarkably coherent scale patterns in all three data sets of Fitts (1954) (see Figures 4, 7, and 11). We have found scale effects of various sizes but invariably of high consistency, with sets of slopes signaling either a $\mathrm{U}$-shaped dependency between $T$ and $S$, as in Exps. 1 and 3, or just one side of this dependency as in Exp. 2.

Task scale deserves consideration in polar analysis for at least two reasons. One is that an undetected effect of this independent variable may interfere with Fitts' law and badly blur the picture (see Figure 1). Another reason is that the scale of movements constitutes in and of itself an important subject in the study of human performance. To illustrate, consider the field of human-computer interaction (HCI), in which considerable research efforts have been dedicated since Card, English, and Burr (1978), using the Fitts paradigm, to the experimental evaluation of the efficiency of pointing devices such as mice, digitizing tablets, trackballs, or touchpads. This research stream has never ceased to hold tight to the incomplete one-DoF polar approach 
inaugurated by Fitts (1954) and researchers have been mostly concerned with the informationtransmission rate issue, remaining essentially unaware of the very existence, at the core of their problem, of an independent variable other than $I D$.

The $T$ vs. scale function tells us how performance gradually drops down as the task is being scaled up and down away from its optimum, and so it is important for designer to realize that the nearer to its optimum the scale of their interface, the better the expected performance. So far the scale issue seems to have been entirely overlooked in the evaluation of input devices in $\mathrm{HCI},{ }^{16}$ but apparently a promising avenue is wide open for research. And one may presume that Fitts' law research has something to learn from the considerable literature that has accumulated, since D'Arcy Thompson's (1942) treatise On Growth and Form, on the relations borne by form and scale in biology (Pennycuick, 1992) and physics (McMahon \& Bonner, 1983).

Perhaps the most rewarding result of the polar chapter of the present study is the discovery that the outcomes of Fitts's three experiments compose a clear-cut three-class taxonomy for classifying the possible outcomes of the Fitts paradigm. Fitts's own data eloquently show that the Fitts time-minimization paradigm may possibly lead to three qualitatively distinct outcomes:

(1) A demonstration of the strong, isochronous form of Fitts' law, as in Fitts's famous Exp. 1 on tapping. Here one obtains just Fitts' law, a lawful relation between $T$ and $F$, with little or no effect of scale, and no $F * S$ interaction.

(2) A demonstration of the weak, non-isochronous form of Fitts' law, as in Fitts's less famous Exp. 2 on disc transfer. Here, the combination of the effects of $F$ and $S$ being additive, one obtains Fitts' law plus an effect of scale.

\footnotetext{
${ }^{16}$ One of the most influential Fitts' law studies in the HCI domain is MacKenzie (1991). In his Exp. 1 the author ran a comparative experimental evaluation of three popular input devices (the mouse, the digitizing tablet, and the trackball) for two aimed-movement tasks (pointing vs. dragging). Fortunately, for one of his six conditions (mouse pointing), MacKenzie reported his data in detailed tabular form (Table 9, p. 87), making it possible to visualize the influence of scale on performance by plotting mean $T$ as a function of $S$, with $F$ (or the $I D$ ) as a parameter, as in Figures 4, 7, and 11 above. As could be expected, the pattern is nicely U-shaped with quite some converging evidence that the optimum was located at about $10 \mathrm{~cm}-\mathrm{a}$ fact of potential relevance in the context under consideration. The effect of scale was small in size and there was no $F * S$ interaction, and so the outcome was essentially the same as that obtained by Fitts in his tapping experiment. Unfortunately, the author did not inquire into the possibility of comparing his three input devices in terms of the performance vs. scale function, and for lack of the appropriate numerical tables there is no way to check.
} 
(3) A violation of Fitts' law, as in Fitts's Exp. 3 on pin transfer, to which the literature has paid so little attention. Here not just the intercept but also the shape of the $T$ vs. $F$ relation varies with the scale level, meaning that the effects of $F$ and $S$ on $T$ combine interactively. One might perhaps attempt to describe the pattern with a two-factor model of the form $T=f(F, S)$, as we did in Section 3.3.1, but the interaction leaves no room for a useful mathematical description of the form $T=f(F)$, thus precluding any conceivable variant of Fitts' law.

It comes as a good surprise that Fitts's results happen to fall unambiguously in these three discrete categories. Interaction, one key criterion of this taxonomy, comes in degrees and so intermediate cases must exist. Nonetheless, with this simple three-class taxonomy in mind, one should be less disarmed in the face of the results of a Fitts' law experiment than Fitts and his successors have traditionally been, often confused by an incomplete understanding of their polar approach. ${ }^{17}$

One puzzling fact about the traditionally polar approach to Fitts' law is what may be called the blind spot of scale. More than sixty years have elapsed since Fitts's discovery of the all-important role played in his time-minimization paradigm by $F$, the quotient of $D / W$. How can it be that mainstream Fitts' law research continues to overlook the possibility of an additive or, worse, interactive effect of $S$ ? How can authors still be content with incomplete graphical representations of their data such as those of Figure 1?

There is reason to speculate that the blind spot of scale extends well outside of the Fitts paradigm. A closely-related case is the spread-minimization paradigm introduced by Schmidt et al. (1979), which uses the same three basic measures of aimed movement as does the Fitts paradigm, but assigns them different statuses. In the Schmidt et al. paradigm experimenters manipulate orthogonally the mean amplitude $\left(\mu_{\mathrm{A}}\right)$ and the mean duration $\left(\mu_{\mathrm{T}}\right)$ of their participants' movements, taking as their dependent measure the spread of movement endpoints $\left(\sigma_{\mathrm{A}}\right.$, the standard deviation of amplitude). There has been a consensus to admit that this paradigm

\footnotetext{
${ }^{17}$ Taking the viewpoint of practitioners of Fitts' law in HCI and holding tight to the one-DoF polar understanding of the Fitts paradigm, Soukoreff and MacKenzie (2004, p. 768) have proposed a conventional criterion, justified by past experience, for deciding whether or not an experiment has corroborated Fitts' law. A normal fit, they suggested, is one with typically $r \geq .9$, meaning $r^{2}=.81$. Using this criterion all three experiments of Fitts (1954) are corroborations of Fitts' law (see Figure 1).
} 
has allowed the demonstration of the Schmidt law, as it is often called, meaning $\sigma_{\mathrm{A} \propto} \mu_{\mathrm{V}}$, where $\mu_{\mathrm{V}}$, the quotient of $\mu_{\mathrm{A}} / \mu_{\mathrm{T}}$, denotes the average speed of the movement. But notice that the Schmidt et al. paradigm, just like the Fitts paradigm, can be construed in Cartesian terms $\sigma_{\mathrm{A}}=f$ $\left(\mu_{\mathrm{A}}, \mu_{\mathrm{T}}\right)$ or just as well in polar terms $\sigma_{\mathrm{A}}=f\left(\mu_{\mathrm{V}}, S\right)$, where scale $S$ is the quantity needed to tell the difference, e.g., for $\mu_{\mathrm{V}}=1 \mathrm{~m} / \mathrm{s}$, between a condition where $5 \mathrm{~cm}$ must be covered in $50 \mathrm{~ms}$ and one where $50 \mathrm{~cm}$ must be covered in 500ms. To this writer's knowledge, students of the Schmidt law have always held tight to an incomplete polar understanding of the Schmidt paradigm, invariably ignored the possible impact of $S$ (Bongers, Fernandez, \& Bootsma, 2009; Schmidt et al., 1979; Wright \& Meyer, 1983; Zelaznik, Shapiro, \& McKolsky, 1981; Zelaznik, Mone, McCabe, \& Thaman, 1988). To realize that it is no less problematic to leave aside the scale dimension in the Schmidt et al. than the Fitts paradigm, consider the example just given: it is very easy for the human arm to satisfy $\mu_{\mathrm{V}}=1 \mathrm{~m} / \mathrm{s}$ by covering $50 \mathrm{~cm}$ in $500 \mathrm{~ms}$ but certainly not $5 \mathrm{~cm}$ in $50 \mathrm{~ms}$ because of acceleration limitations. Here again, just as in the Fitts paradigm, one must hypothesize some optimal function, the next question being of course how $F$ (i.e., $\mu_{\mathrm{v}}$ ) and $S$ (e.g., $\mu_{\mathrm{A}}$ ) combine their effects on endpoint spread.

Many illustrations could be given of how difficult it is to bear in mind that the calculation of a quotient automatically sacrifices one DoF. As already noted, human performance often needs to be evaluated in terms of both its speed and its accuracy and a variety of data compression techniques have been devised to combine these two dimensions of performance. One instance is the so-called throughput, possibly computed as the quotient of $I D$ by $T$, often used by Fitts' law experimenters in HCI (ISO, 2000; Soukoreff \& MacKenzie, 2004). The throughput being a measure of performance that combines speed and accuracy, it is tempting to forget that that oneDoF measure cannot characterize the performance in full. However impressive a given throughput score, there is no warrantee that the performance is good, simply because a very high throughput may well be associated with, say, an unacceptably low level of accuracy.

A telling illustration can be found in the aiming performance of our saccadic ocular system. There is considerable converging evidence that that system is both much more efficient and much more hasty than the hand system (Harris \& Wolpert, 1998). No matter whether one calculates a throughput, or a resource as in the WHo model of Guiard and Rioul (2015), or whatever global performance index, the capability of the eye system to reach its targets in the 
visual field is peerless, and this is easy to explain given its massive recourse to hard-wired neural circuitry. However, judging by the coefficient of variation $\left(\sigma_{\mathrm{A}} / \mu_{\mathrm{A}}\right)$ of saccade amplitude, which measures relative error, the aiming behavior of the ocular system is very inaccurate indeed, in comparison with that of the hand system. ${ }^{18}$ The point being made is that from the moment speed and accuracy are compressed into one polar DoF, another DoF must be considered to obtain a complete description of the performance.

To finish with a mundane analogy, it is no better justified to ignore $S$ in the usual polar understanding of the Fitts paradigm than it is to ignore the quality of a product whose quality/price ratio is known. Assuming the ratio is, say, exceptionally high, the customer still cannot take a decision before learning about the other half of the story, whether made up of price or quality information.
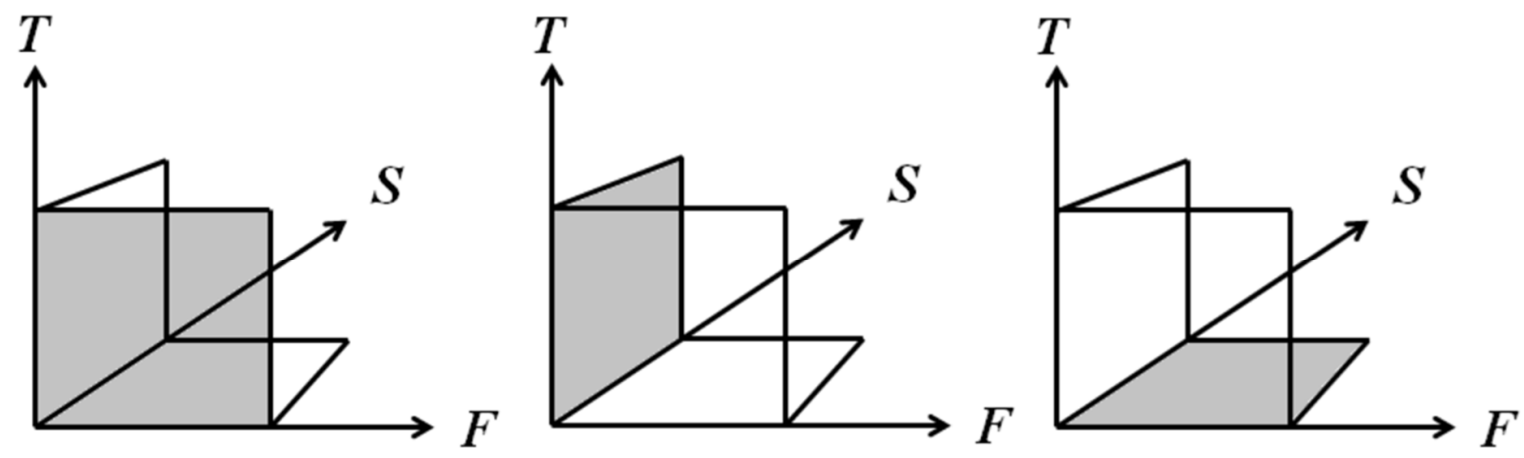

Figure 14. The three planes of the Fitts paradigm, according to the complete polar representation, with the Fitts' law plane shown on the front, the scale effect plane on the left, and the experimental design plane on the ground.

In fact two definite recommendation have arisen from this study. One is that if for whatever reason one is choosing the polar description of the Fitts paradigm, or of a similar paradigm, then one should make sure that description is complete, involving both $F$ and $S$. To

\footnotetext{
${ }^{18}$ Revisiting the data of Abrams, Meyer and Kornblum (1989), Guiard (in preparation) has found that the coefficient of variation of the saccade to visual targets ranges from about $10 \%$ up to about $25 \%$. These values should be compared for example with the 0\%-12\% range found by Guiard, Olafsdottir and Perrault (2011) in a hand-movement experiment especially designed to encourage their participants to explore their full spectrum of speed/accuracy strategies.
} 
avoid confusions about Fitts' law-e.g., between its strong and weak version-reasoning needs to take place in the 3D space of Figure 14.

The other main lesson from this study is that there may be simple structure in the alternative, less familiar Cartesian description of the same data, as illustrated by the data of Fitts's pin-transfer experiment. Having found a disappointingly complex (i.e., interactive) pattern in polar space, we discovered that the pattern was very simple indeed (i.e., neatly additive) in Cartesian space.

That sort of result is somewhat disquieting for the realist, who takes it as an axiom that the world is what it is independently of our theories and beliefs about it. How can it be that the Fitts paradigm, which is so simple, suffers a sort of conceptual bi-stability reminiscent of the perceptual bi-stability of the Necker cube? One possibility worth considering is that the timeminimization paradigm of Fitts is too simple. Recall J. J. Gibson's (1979) critique of traditional studies of perception. Most illusions - the Necker cube is one instance among many-are mere laboratory artefacts due to the fact that the information delivered to the senses is dramatically impoverished.

Philosopher of science Susan Haack (2009) proposed that we progress in scientific research in pretty much the same way as we do in solving crossword puzzles (see Figure 15). The main motivation of the crossword model is that it "permits pervasive mutual support rather than, like the model of a mathematical proof, encouraging an essentially one-directional conception" (Haack, 2009, p. 126).

In fact the task that students of simple aimed movement have assigned to themselves is not really like a crossword puzzle because they have not imposed on themselves the constraint of cross-checked coherence. Strictly the same three quantities being considered-movement amplitude, movement error, and movement time-there has been a stable consensus in the literature that the law is nonlinear (Fitts' law) if the dependent measure is movement time, but linear (Schmidt's law) if the dependent measure is endpoint spread. Put differently, it has been generally accepted that the model $y=f(x)$ being assumed to be true, the model $x=f^{-1}(y)$ can be false. 

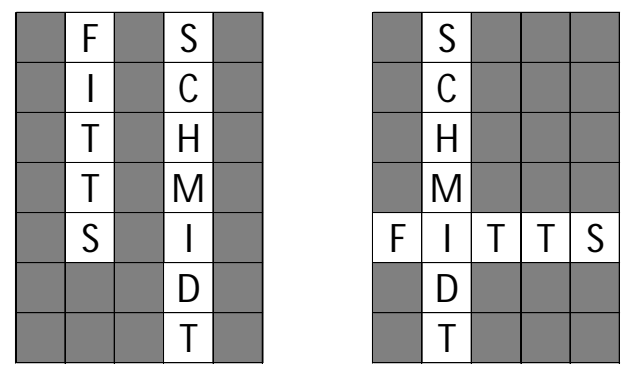

Figure 15. Haack's crossword puzzle metaphor.

This is like treating the problem at hand as though it consisted of two separate, unrelated questions. One important property of crossword puzzles is that the more intricate their structure, the less ambiguity in the solution. As emphasized by Atkinson and Peijnenburg (2010), the puzzle needs to be sufficiently complex to have a unique solution. One characteristic of strong science is that it relies on not just solid measurement and observation, but also converging evidence.

\section{References}

Abrams, R. A., Meyer, D. E., \& Kornblum, S. (1989). Speed and accuracy of saccadic eye movements: characteristics of impulse variability in the oculomotor system. Journal of Experimental Psychology: Human Perception and Performance, 15(3), 529.

Accot, J., \& Zhai, S. (2001). Scale effects in steering law tasks. In Proceedings of the SIGCHI conference on Human factors in computing systems (pp. 1-8). ACM.

Akaike, H. (1973). Information theory and an extension of the maximum likelihood principle. In Petrov, B. N. \& Csáki, F. (Eds.), Second International Symposium on Information Theory (pp. 267-281). Budapest: Akadémiai Kiadó.

Annett, J., Golby, C. W., \& Kay, H. (1958). The measurement of elements in an assembly task: The information output of the human motor system. Quarterly Journal of Experimental Psychology, 10(1), 1-11. 
Atkinson, D., \& Peijnenburg, J. (2010). Crosswords and coherence. Review of Metaphysics, 63, 807-820.

Beamish, D., Bhatti, S. A., MacKenzie, I. S., \& Wu, J. (2006). Fifty years later: a neurodynamic explanation of Fitts' law. Journal of the Royal Society Interfaces, 3, 649-654.

Bongers, R., Fernandez, L., \& Bootsma, R. J. (2009). Linear and logarithmic speed-accuracy trade-offs in reciprocal aiming result from task-specific parameterization of an invariant underlying dynamics. Journal of Experimental Psychology: Human Perception and Performance, 35, 1443-1457.

Card, S. K., English, W. K., \& Burr, B. J. (1978). Evaluation of mouse, rate-controlled isometric joystick, step keys, and text keys for text selection on a CRT. Ergonomics, 21, 601-613.

Crossman, E. R. F. W. (1956). The Measurement of Perceptual Load in Manual Operations. Unpublished Ph. D. thesis. Birmingham University.

Crossman, E. R. F. W., \& Goodeve, P. J. (1983). Feedback control of hand-movement and Fitts' law. The Quarterly Journal of Experimental Psychology, 35A, 251-278.

Elliott, D., Helsen, W. F., \& Chua, R. (2001). A century later: Woodworth's two-component model of goal-directed aiming. Psychological Bulletin, 127, 342-357.

Fitts, P. M. (1953). The influence of response coding on performance in motor tasks. Current Trends in Information Theory. University of Pittsburgh Press, Pittsburgh, PA, 47-75.

Fitts, P. M. (1954). The information capacity of the human motor system in controlling the amplitude of movement. Journal of experimental psychology, 47, 381. Republished in 1992 in the Journal of Experimental Psychology: General, 121, 262-269.

Fitts, P. M., \& Peterson, J. R. (1964). Information capacity of discrete motor responses. Journal of experimental psychology, 67, 103.

Flach, J. M., Guisinger, M. A., \& Robison, A. B. (1996). Fitts's Law: Nonlinear Dynamics and Positive Entropy. Ecological Psychology, 8, 281-325. 
Furnas, G. W., \& Bederson, B. B. (1995). Space-scale diagrams: Understanding multiscale interfaces. In Proceedings of the SIGCHI conference on Human factors in computing systems (pp. 234-241). ACM Press/Addison-Wesley Publishing Co.

Gibbs, C. B. (1962). Controller design: Interactions of controlling limbs, time-lags, and gains in positional and velocity systems. Ergonomics, 5, 385-402.

Gibson, J. J. (1979). The Ecological Approach to Visual Perception. Hove (UK): Psychology Press.

Gori, J., Rioul, O., \& Guiard, Y. (2018). Speed-Accuracy Tradeoff: A Formal InformationTheoretic Transmission Scheme (FITTS). ACM Transactions on Computer-Human Interaction, 25 (5).

Gori, J., Rioul, O., Guiard, Y., \& Beaudouin-Lafon, M. (2018). The Perils of Confounding Factors: How Fitts' Law Experiments can Lead to False Conclusions. In Proceedings of the 2018 CHI Conference on Human Factors in Computing Systems (p. 196). ACM.

Guiard, Y. (1997). Fitts' law in the discrete vs. cyclical paradigm. Human Movement Science, 16, 97-131.

Guiard, Y., \& Olafsdottir, H. B. (2011). On the measurement of movement difficulty in the standard approach to Fitts' law. PLoS one, 6(10), e24389.

Guiard, Y., Olafsdottir, H. B., \& Perrault, S. T. (2011). Fitts' law as an explicit time/error tradeoff. In Proceedings of the SIGCHI Conference on Human Factors in Computing Systems (pp. 1619-1628). ACM.

Guiard, Y., \& Rioul, O. (2015). A mathematical description of the speed/accuracy trade-off of aimed movement. Proceedings of the 2015 British HCI Conference (pp. 91-100). ACM.

Haack, S. (1993/2009). Evidence and Inquiry: A Pragmatist Reconstruction of Epistemology (2 $2^{\text {nd }}$ Edition). Amherst (NY): Prometheus Books.

Harris, C. M., \& Wolpert, D. M. (1998). Signal-dependent noise determines motor planning. Nature, 394, 780-784. 
Hess, R. A. (1973). Nonadjectival rating scales in human response experiments. Human Factors, $13,275-280$.

Hick, W. E. (1952). On the rate of gain of information. Quarterly Journal of Experimental Psychology, 4, 11-26.

Hoffmann, E. R. (2013). Which version/variation of Fitts' law? A critique of information-theory models. Journal of motor behavior, 45, 205-215.

ISO/DIS 9241-9 Ergonomic requirements for office work with visual display terminals (VDTs) Part 9: Requirements for non-keyboard input devices. International Standard, International Organization for Standardization (2000).

Keele, S. W. (1968). Movement control in skilled motor performance. Psychological bulletin, 70, 387-403.

Kelso, J. A. S. (1992). Theoretical concepts and strategies for understanding perceptual-motor skills: From information capacity in closed systems to self-organization in open, nonequilibrium systems. Journal of Experimental Psychology: General 121, 260-261.

Kvålseth, T. O. (1980). An alternative to Fitts' law. Bulletin of the psychonomic Society, 16, 371373.

Kuemmerle, W. (1998). Optimal scale for research and development in foreign environments: An investigation into size and performance of research and development laboratories abroad. Research Policy, 27, 111-126.

Luce, R. D. (1985). Response Times and their role in inferring elementary mental organization. New York: Oxford University Press.

Luce, R. D. (1986). Response time distributions in memory search: A caution. In: F. Klix and H. Hagendorf (Eds), Mechanisms and Performances, pp. 109-121. Amsterdam: North-Holland.

Luce, R. D. (2003). Whatever happened to information theory in psychology? Review of General Psychology, 7, 183-188. 
MacKenzie, I. S. (1991). Fitts' law as a Performance Model in Human-Computer Interaction. Doctoral dissertation. University of Toronto. ISBN 0315659858

MacKenzie, I. S. (1992). Fitts' law as a research and design tool in human-computer interaction. Human-Computer Interaction, 7, 91-139.

McMahon, T. A., \& Bonner, J. T. (1983). On Size and Life. New York: Scientific American Library. $b$

Meyer, D. E., Abrams, R. A., Kornblum, S., Wright, C. E., \& Smith, J. E. K. (1988). Optimality in human motor performance: Ideal control of rapid aimed movements. Psychological Review, 95, 340-370.

Mottet, D., \& Bootsma, R. J. (1999). The dynamics of goal-directed rhythmical aiming. Biological cybernetics, 80, 235-245.

Pachella, R. G. (1973). The interpretation of reaction time in information processing research. Technical report No. TR-45. Michigan University Ann Arbor Human Performance Center.

Pennycuick, C. J. (1992). Newton Rules Biology - A Physical Approach to Biological Problems. Oxford (UK): Oxford University Press.

Plamondon, R., \& Alimi, A. M. (1997). Speed/accuracy trade-offs in target-directed movements. The Behavioral and Brain Sciences, 20, 279-303.

Schmidt, R. A., Zelaznik, H. N., Hawkins, B., Frank, J. S., \& Quinn, J. T. (1979). Motor-output variability: A theory for the accuracy of rapid motor acts. Psychological Review, 86, 415-451.

Shannon, C. (1948). A mathematical theory of communication. Bell System Technical Journal, 27, 379-423, 623-656.

Sheridan, M. R. (1979). A reappraisal of Fitts' Law. Journal of Motor Behavior, 11(3), 179-188.

Solomonoff, R. J. (1964). A formal theory of inductive inference. Information and Control,

7, 1-22. 
Soukoreff, R. W., \& MacKenzie, I. S. (2004). Towards a standard for pointing device evaluation, perspectives on 27 years of Fitts' law research in HCI. International Journal of HumanComputer Studies, 61, 751-789.

Thompson, D. W. (1942/1992). On Growth and Form: A New Edition. Cambridge (UK): Cambridge University Press.

Viviani, P., \& Terzuolo, C. (1982). Trajectory determines movement dynamics. Neuroscience, 7 , 431-437.

Wickelgren, W. A. (1977). Speed-accuracy tradeoff and information processing dynamics. Acta psychologica, 41, 67-85.

Wiener, N. (1948). Cybernetics: Control and Communication in the Animal and the Machine. New York: Wiley.

Woodworth, R. S. (1899). Accuracy of voluntary movement. Psychological Review: Monograph Supplements, 3(3).

Wright, C. E., \& Meyer, D. E. (1983). Conditions for a linear speed-accuracy trade-off in aimed movements. Quarterly Journal of Experimental Psychology, 35A, 279-296.

Zelaznik, H. N., Shapiro, D. C., \& McKolsky, D. (1981). Effect of a secondary task on the accuracy of single-aiming movements. Journal of Experimental Psychology: Human Perception and Performance, 7, 1007-1018.

Zelaznik, H. N., Mone, S., McCabe, G. P., \& Thaman, C. (1988). Role of temporal and spatial precision in determining the nature of the speed/accuracy trade-off in aimed hand movements. Journal of Experimental Psychology: Human Perception and Performance, 14, 221-230.

Zhai, S. (2004). Characterizing computer input with Fitts' law parameters - the information and non-information aspects of pointing. International Journal of Human-Computer Studies, 61, 791-809. 